\title{
The composite Triassic-Eocene Poshteh pluton, eastern Iran, an Eo-Cimmerian element south of the main Paleotethys suture
}

\author{
Shahryar Mahmoudi ${ }^{1}$ (D) Fernando Corfu $^{2}$ (D)
}

Received: 16 November 2021 / Accepted: 19 January 2022 / Published online: 5 February 2022

(c) The Author(s) 2022

\begin{abstract}
The composite Poshteh Pluton, at the northeastern margin of the Central Iranian Microplate near Taybad in eastern Iran, is positioned at a critical tectonic junction, south of the inferred main Paleotethys suture and along the major regional Doruneh Fault system. It consists of two distinct intrusions. Quartz monzonite is dated in this study by zircon U-Pb ID-TIMS to 215.8 $\pm 0.5 \mathrm{Ma}$, an age that coincides with the time of closure of the Paleotethys during the late collisional stages of the EoCimmerian Orogeny. It is geochemically very similar to coeval plutons present along and north of the Paleotethys suture, where they intruded Carboniferous-Permian arc sequences, ophiolites and flysch. The Poshteh quartz monzonite is located south of the suture in a position similar to the Anarak and related complexes further west, which previously have been interpreted as reflecting Mesozoic and Cenozoic disruption of the Eo-Cimmerian Orogen by extensional and transtensional processes. The Triassic quartz monzonite was subsequently invaded by granite at $41.23 \pm 0.31 \mathrm{Ma}$. The emplacement was in part structurally controlled by the Doruneh Fault system and associated to hydrothermal alteration and Fe mineralization. The granite is thus a coeval member of a widespread late Eocene to Oligocene plutonic suite in the region, and likely the result of delamination and melting of the subcontinental lithosphere.
\end{abstract}

Keywords Eo-Cimmerian Orogeny $\cdot$ Paleotethys $\cdot$ Eocene $\cdot$ Pluton $\cdot$ Zircon $\cdot \mathrm{U}-\mathrm{Pb}$

\section{Introduction}

Eastern Iran consists of a complex collage of tectonic elements. It includes late Precambrian to early Paleozoic terranes, derived from northern Gondwana, and variously affected, and eventually welded together, by two major orogenic cycles. The first in the Paleozoic, ending with the Triassic Eo-Cimmerian Orogeny, and the second in the Cenozoic corresponding to the Alpine Orogeny (e.g. Stöcklin 1977; Berberian and King 1981; Sengör 1984; Besse et al. 1998; Golonka 2004; Zanchi et al. 2009a). The late Precambrian processes involved mainly magmatic growth along the northern margin of Gondwana. Early Paleozoic rifting

Fernando Corfu

fernando.corfu@geo.uio.no

Shahryar Mahmoudi

S.mahmoudi@khu.ac.ir; Shahryar.mahmoudi@gmail.com

1 Faculty of Earth Sciences, Geochemistry Department, Kharazmi University, Tehran, Iran

2 Department of Geosciences and CEED, University of Oslo, Blindern, Postbox 1047, 0316 Oslo, Norway detached ribbon terranes and opened the Rheic Ocean (e.g. Stampfli and Borel 2002; Bagheri and Stampfli 2008; Torsvik and Cocks 2013; Domeier 2018; Ranjbar Moghadam et al. 2018). A subsequent stage of rifting in the Devonian caused deposition of clastic sediments and evaporites and was followed by marine sedimentation, gradually opening the Paleotethys (Bagheri and Stampfli 2008). This opening preceded diachronously from west to east, starting as back-arcs related to subduction of the Rheic Ocean plate, and led to the separation from northern Gondwana of ribbon microcontinents (Galatian superterrane; Stampfli et al. 2013). Subduction of the Paleotethys plate started in the middle to late Devonian and continued through the Carboniferous, as recorded by ophiolites, by arc magmatism, and metamorphic stages at the margin of the Turan (Eurasian) continental plate (Ruttner 1993; Bagheri and Stampfli 2008; Zanchetta et al. 2009, 2013; Shafaii Moghadam et al. 2015a). By the middle Triassic the Paleotethys plate was eventually completely consumed once the Cimmerian block, which had separated from Gondwana during the opening of the Neotethys in the Permian, collided with the Eurasian plate: this is referred to as the Eo-Cimmerian Orogeny. Late Triassic 
to early Jurassic molasse deposits of the Shemshak Group define the final stages of this orogenic event (Zanchi et al. 2009a; Wilmsen et al. 2009). In Iran and Afghanistan the Paleotethys suture can be tracked mostly along the southern margin of the Turan Plate, but subsequent tectonic processes have contributed to its disruption, and some parts are now found south of the main suture. Of particular importance for the present paper are the relationships at the northern margin of the Central Iranian Microplate (CIM) south of the Doruneh Fault. Here the Anarak, Jandaq and Posht-e-Badam metamorphic complexes (Fig. 1) record several stages of the Paleotethys convergence and subduction history, including ophiolites and flysch deposits, late Carboniferous-early Permian metamorphism, and the final late Triassic EoCimmerian collision (Fig. 1; Bagheri and Stampfli, 2008; Zanchi et al. 2009b, 2015).

The Neotethys had opened in the late Carboniferous to Permian (Stampfli et al. 2013) and eventually started to contract. In the Cenozoic it closed as a consequence of convergence and collision of Arabia with Central Iran. The tectonic processes in Iran were also directly affected by the larger scale convergence of India with Asia and the resulting lateral indentation (e.g. Gaina et al. 2015; Bagheri and Gol 2020). The interaction of the various tectonic elements through time has resulted in the very complex geological relationships we observe today.

The present study is focused on the composite Poshteh Pluton in eastern Iran, the product of two distinct magmatic events, the first in the Triassic and the second in the Eocene. This pluton is hosted by a metamorphosed supracrustal sequence and was originally mapped as an entirely Eocene intrusion (Ternet et al. 1980). The present documentation of the original Triassic history has special relevance for the paleogeography and the processes related to the closure of the Paleotethys and the Eo-Cimmerian

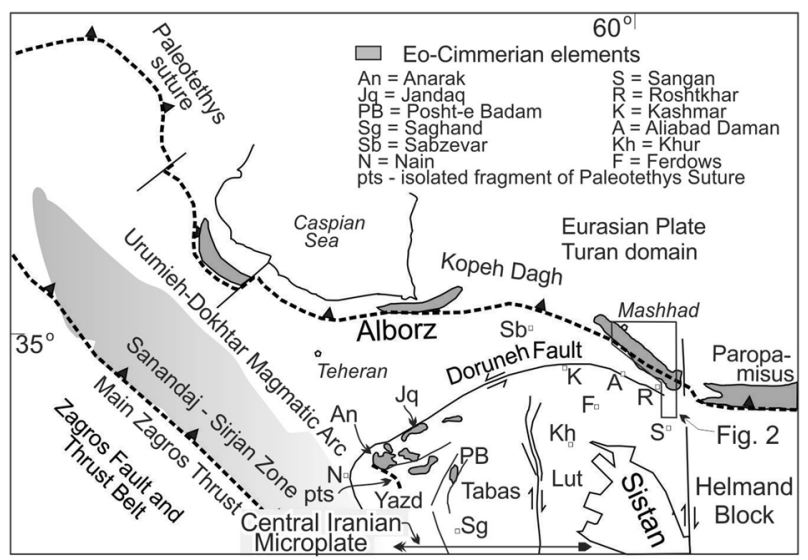

Fig. 1 Simplified tectonic map of Iran with location of units mentioned in text
Orogeny and the subsequent Cretaceous and Paleogene to recent tectonics in the region. An important question concerns the location of this Triassic intrusion to the south of the main Paleotethys suture, that mimics other occurrences further west, but also very similar in age and chemical composition to several coeval plutons north of the main Paleotethys suture zone (Figs. 1, 2). The Eocene granite corresponds instead to a very widespread magmatic province in eastern Iran. The paper documents the ages and the main compositional features of the distinct intrusions and discusses their regional tectonic significance in the context of the known tectonic framework.

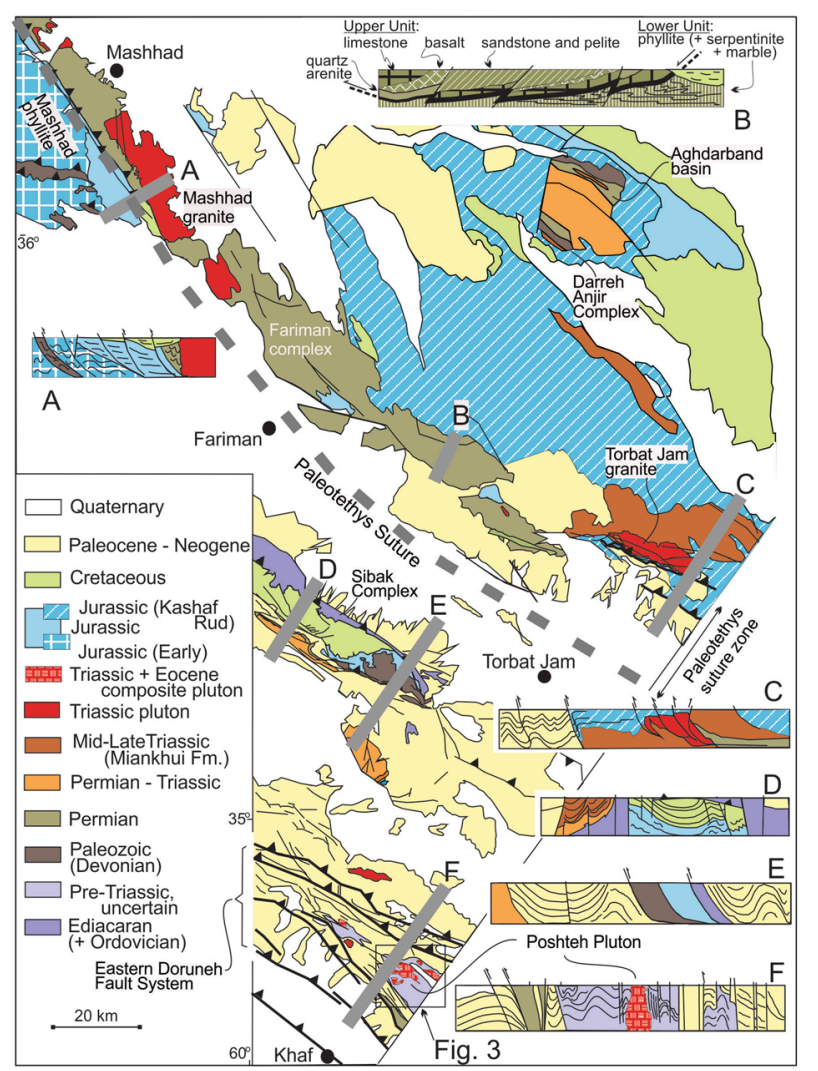

Fig. 2 Simplified map and cross sections of the region between Mashhad and Khaf. The cross sections are not at scale and idealized to reflect the main relationships and structures in the region. The map and cross sections integrate information from de Gramont et al. (1984), Ternet et al. (1980), Alavi-Naini and Mossavi-Khorzughi (2006), Farbod et al. (2011), Sheikholeslami and Kouhpeyma (2012), Zanchetta et al. (2013), Javadi et al. (2013), Zanchi et al. (2016) and Ranjbar Moghadam et al. (2018) 


\section{Geological setting}

\section{Regional framework}

The dominant geological elements of eastern Iran are the CIM and the Helmand (Afghan) block, which are separated by the Sistan suture zone (Eastern Iranian Orogen), and are succeeded farther north by a series of belts of different origins and ages (Fig. 1; Bagheri and Gol 2020). The CIM consists of the Lut, Tabas and Yazd blocks, which are separated from each other by a series of broadly $\mathrm{N}-\mathrm{S}$ trending faults. The oldest units of the CIM are Ediacaran to Cambrian (e.g. Ramezani and Tucker 2003; Bagheri and Stampfli 2008), but they were variously affected by subsequent orogenies, especially in connection with the Devonian to Triassic Paleotethys events, and subsequently during several Mesozoic and Cenozoic orogenic stages.

The CIM is bound to the north by the Doruneh Fault system, which has a multistage kinematic history. It acted as a dextral system in response to NW-SE transpression during the Eocene and Miocene, but later became a leftlateral system controlled by the anticlockwise rotation of the CIM (Javadi et al. 2013) and N-S compression (Mattei et al. 2012; Tadayon et al. 2017, 2019). Bagheri and Gol (2020) propose that the indentation of India into Eurasia played a role on the kinematics of these faults.

\section{Geology across the Paleotethys suture south of Mashhad}

Figure 2 displays the broad geological relationships across the Paleotethys suture in the region south of Mashhad in eastern Iran. A series of idealized cross sections illustrate the main structural relationships. The general structural grain is dominated by SE trending ridges, with exposures of pre-Jurassic rocks along the ridges, separated by bands of late Mesozoic and younger rocks with many faults and thrust faults, dominantly with top to the SW kinematics. These faults generally affect also the youngest Cenozoic strata, and are thus very young structures, but some are older and related to the various Mesozoic events that affected the region, especially the late Triassic Eo-Cimmerian Orogeny.

Important evidence for the Paleotethys suture are the ophiolitic sequences preserved at Mashhad, the Fariman Complex and the Darreh Anji Complex (Fig. 2). The latter comprises a section of Devonian (ca. $380 \mathrm{Ma}$ ) gabbro and minor ultramafic rocks together with local felsic intrusives, which have been interpreted to indicate subduction initiation (Shafaii Moghadam et al. 2015a). The gabbros are thrust on a sequence of basaltic lava flows with marble lenses and intercalations of radiolarian chert of Permian age (Zanchetta et al. (2013). The Fariman Complex consists of a lower unit of micaschists and calcareous phyllites with some meta-basalts, marbles and serpentinite, and an upper unit including Permian limestone and basaltic to andesitic lava flows interfingering with volcanoclastics and clastic sedimentary rocks (Profile B in Fig. 2; Zanchetta et al. 2013). The rocks are Permian and the sheared contact between the two units predates deposition of the Kashaf Rud Formation in the Mid-Jurassic (Zanchetta et al. 2013). The latter authors suggest a back-arc origin of the Fariman complex. By contrast, Topuz et al. (2018), based on a geochemical investigation of basaltic rocks, concluded that the Fariman Complex originated by melting of a mantle plume and interpret it as a 'fragment of an oceanic plateau, which escaped subduction and was accreted as exotic block in the Paleotethys suture zone.' Ultramafic rocks, gabbrodiorite and pillow basalt associated with chert, marble and turbiditic rocks characterize the northern extension of this complex at Mashhad (Shafaii Moghadam et al. 2015a). The assemblage also includes a flysch sequence of deep sea turbidites which was interpreted as an accretionary prism by Alavi (1991).

Near Mashhad the above unit is unconformably overlain by the late Triassic-early Jurassic 'Mashhad Phyllite' (Fig. 2), which is considered the equivalent of the regionally widespread lower Shemshak Group (Sheikholeslami and Kouhpeyma 2012). These rocks were then covered unconformably by Jurassic clastic deposits, in part equivalent to the Kashaf Rud Formation, and subsequently by Cretaceous sediments.

In the Aghdarband Basin, late Triassic arc-related marine sediments (Sina Formation) were covered by continental deposits of the Miankuhi Formation (Ruttner 1993; Zanchi et al. 2016; Mazaheri-Johari et al. 2021, 2022). The Miankuhi Formation was deformed and intruded by the Torbat Jam granite at $217 \pm 1.8 \mathrm{Ma}$ (Zanchetta et al. 2013). The whole sequence was then covered by the late to post-tectonic Kashaf-Rud Formation. The Torbat Jam granite is part of a coeval suite of granitoid plutons intruding the Mashhad ophiolite between 217 and $200 \mathrm{Ma}$ (Karimpour et al. 2010; Mirnejad et al. 2013; Deyhimi et al. 2019).

South of Fariman and west of Torbat Jam a series of basement units have been upthrust through the Cenozoic cover. These units include the Sibak Complex of Ediacaran metamorphic and intrusive rocks, locally cut by Ordovician gabbro (Ranjbar Moghadam et al. 2018), in addition to fragments of Devonian, Permian and Triassic units. The locally extensive Cretaceous strata in this area represent the eastern extension of the Sabzevar ophiolitic sequences, which developed in an internal ocean basin opened in this period (Rossetti et al. 2010; Shafaii Moghadam et al. 2014).

The southernmost ridge shown on the map of Fig. 2 comprises some fragments of older metasediments and 
sparse intrusives, which are the main subject of the present study. The main structural grain in the area trends to the SE and was likely controlled by the Doruneh Fault system, which in this area widens into a fan of multiple NE-dipping reverse faults (Farbod et al. 2011).

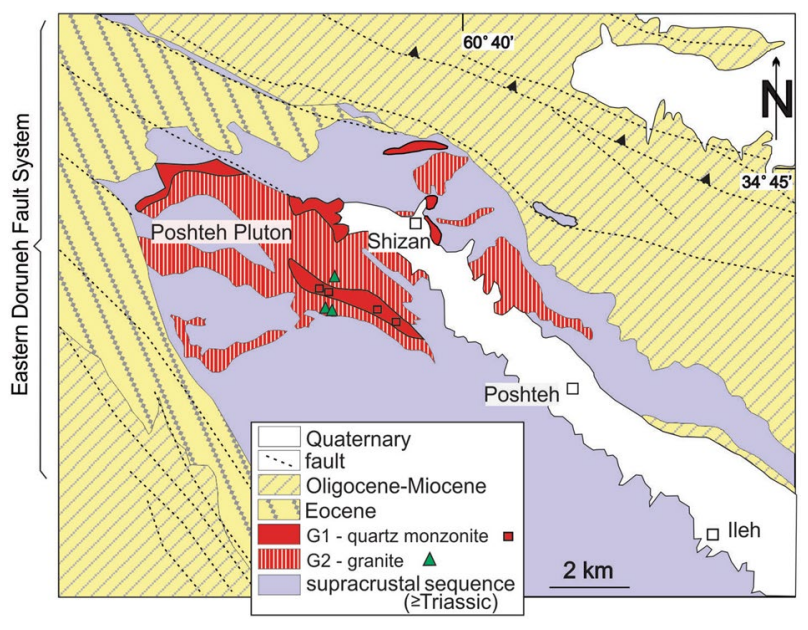

Fig. 3 Geological map of the Poshteh Pluton area (modified from Ternet et al. (1980)). In this area the Doruneh Fault System weidens into a fan of subsidiary faults (Farbod et al. (2011), Javadi et al. (2013))

\section{Setting and country rocks of the Posteh Pluton}

The study area is located at the northeastern edge of the CIM, in the Khorasan Razavi Province (Figs. 1, 2). It consists of a metamorphosed volcano-sedimentary sequence, intruded by the Poshteh Pluton, and surrounded/covered by Eocene-Miocene sedimentary and volcanic assemblages (Figs. 2, 3).

The supracrustal sequence hosting the Poshteh Pluton is non-fossiliferous. Based on regional considerations it was interpreted by Ternet et al. (1980) to be late Precambrian, but presently there is no direct evidence that would permit to verify this hypothesis. Another fault-bounded metasedimentary package further south contains sparse fossils indicating a Devonian age, but the sequence is lithologically different from that at Poshteh (Ternet et al. 1980). The supracrustal rocks were subjected to regional metamorphism, which reached greenschist facies, with higher metamorphic grades superimposed along the contact metamorphic aureole of the Poshteh Pluton (Ternet et al. 1980).

The metavolcanic rocks are mainly mafic and include meta-andesites and meta-basalts, with local amphibolites in the vicinity of the intrusion (Fig. 4b; Ternet et al. 1980). These rocks commonly occur as massive units, in part with thin layers or small green and greenish-grey pods. Primary textures are visible locally, with plagioclase as the major mineral, mostly replaced by secondary minerals. Other minerals are amphibole and pyroxene, pseudomorphically replaced by mica. Metamorphic minerals, including chlorite
Fig. 4 a Granite (G2) intruding quartz monzonite (G1) in the Poshteh Pluton. b Contact of Poshteh Pluton with amphibolite country rocks. c Typical mineralogy of monzonite G1: Plagioclase, grey because of alteration, dominates over biotite (brown) and quartz (white); $\mathrm{K}$-feldspar is not in the picture. d Typical minerals in granite G2: quartz (white, left-upper corner) and feldspar (grey from alteration) near hornblende that has small inclusions of apatite (white) and biotite (brown). Width of picture in $\mathbf{c}$ and $\mathbf{d}$ is about $1 \mathrm{~mm}$
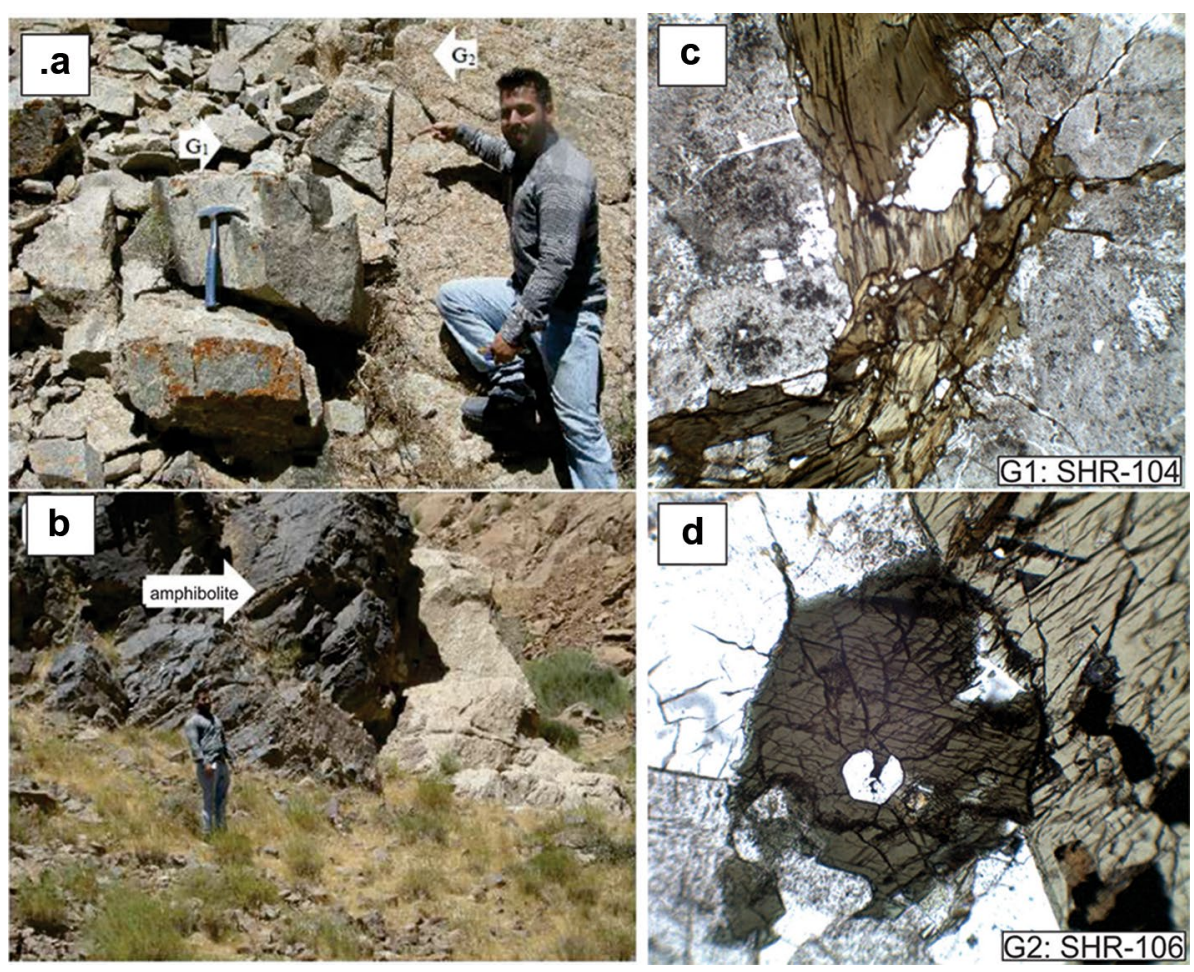
and epidote mostly replacing plagioclase and amphiboles, fill the vesicles in meta-basites and in crosscutting veins.

The metasedimentary rocks consist of mica-schist, marble, quartzite and conglomerate. The main components are silica-rich metapelites with interlayers of marble. The metapelites occur as lenses and thin layers with alternating bands dominated by quartz or by mica. Quartz and feldspar are completely recrystallized into a granoblastic texture oriented parallel to the foliation. The micaceous layers consist mainly of muscovite and some biotite with a lepidogranoblastic texture. There are pseudomorphs, possibly after garnet.

In the marbles and calc-silicates the minerals are mostly oriented (to the NW) and have two different grain sizes, fine- to medium-grained and coarse-grained. Quartz and feldspar are common but they are mostly fine-grained. Calcite, opaques and phlogopite are the major minerals, together with accessory quartz, olivine and amphibole. Large calcite crystals contain silicate inclusions, such as muscovite and pyroxene. White mica is generally oriented and locally surrounds calcite crystals with complex orientations.

\section{The Poshteh Pluton}

\section{Lithologies and mineral compositions}

The Poshteh Pluton is composed of two distinct intrusive units: older quartz monzonite (G1) and younger granite (G2) (Figs. 3, 4a).

The quartz monzonite (G1) consists of plagioclase (60\%), K-feldspar (20-25\%), quartz (5-10\%), biotite (10\%) and accessory zircon and apatite (Fig. 4c). Plagioclase is zoned, poly-synthetically twinned, and highly altered. The rock is approximately equigranular and locally undeformed away from the sheared domains.

Granite $\mathrm{G} 2$ is equigranular and massive. It consists of $\mathrm{K}$-feldspar and plagioclase in amounts visually estimated at 50 and $20 \%$, respectively, but the $\mathrm{K}$-feldspar is perthitic and highly altered so that the exact proportions are difficult to determine. The norm suggests $30 \%$ orthoclase and $40 \%$ plagioclase. Quartz (about 30\%) occurs as individual euhedral to subhedral grains frequently in association with clusters of green-brown hornblende (10\%) and traces of brown biotite (Fig. 4d). Accessory minerals include apatite, epidote, zircon and ore minerals.

Dykes of granite G2, intruded into quartz monzonite G1, are oriented parallel to the NW-trending regional faults indicating a structural control during emplacement, but granite G2 is itself locally deformed, witnessing late reactivation stages. The shear zones are marked by a variety of mylonitic rocks ranging from protomylonitic to non-foliated cataclastic rocks, up to $100 \mathrm{~m}$ wide. Low-grade metamorphism in the shear zone formed sericite and chlorite along S-C structures.
Hydrothermal activity was associated with this shear zone when it was still at depth.

Small bodies of iron ore occur in the contact metamorphic aureoles of the pluton with the marbles and metavolcanic rocks. The geometry of these bodies suggests a link to the emplacement of the granite by hydrothermal metasomatism. However, iron ore veins also cut the overlying Miocene sedimentary cover, possibly reflecting late remobilization. Based on a study of fluid inclusions in the iron deposit Karimi et al. (2012) suggested an origin of the ore from hydrothermal fluids released during granite emplacement, mixing with meteoric water and precipitation during cooling.

\section{Mineral chemistry}

Chemical analyses of minerals were carried out using the CAMECA-SX-100 electron microprobe at the Iran mineral processing research center. Natural and synthetic oxides and silicate standards were used. Operating conditions were $12 \mathrm{kV}$ and $15 \mathrm{nA}$ with a counting time of usually $15 \mathrm{~s}$. Biotite and plagioclase in the quartz monzonite (G1) (sample TR-117), and in the granite (G2) (TR-103) have been analyzed (totally 353 points, Table 1 ).

Biotite in both samples is primary magmatic (Fig. 5a). It has a Fe-rich composition with $\mathrm{XFe}=\mathrm{Fe}^{2+}$ / $\left.\mathrm{Fe}^{2+}+\mathrm{Mg}\right)=0.75-0.1$ and is more ferroan than magnesian (Tischendorf et al. 1997) (Fig. 5b). Biotite in G2 contains 2.26-2.96 wt $\% \mathrm{TiO}_{2}$ (and so its colours change from brown to light brown. Crystallization pressures and temperatures of biotite in granitic magma can be estimated using the empirical geothermometer of Henry et al. (2005), for which the uncertainties are $<300{ }^{\circ} \mathrm{C}$, and the Al-in-biotite geobarometer of Uchida et al. (2007), for which the uncertainties are $<1 \mathrm{kbar}$. The calculated temperature for G2 ranges between 652 and $674{ }^{\circ} \mathrm{C}$ (average $669 \pm 6{ }^{\circ} \mathrm{C}(1 \sigma)$, and the pressure between 2.6 and $3.1 \mathrm{kbar}$ (average $2.9 \pm 0.2 \mathrm{kbar}$ $(1 \sigma)$. Biotite in G1 indicates $682-694{ }^{\circ} \mathrm{C}$ (average $688 \pm 8^{\circ} \mathrm{C}$ $(1 \sigma)$ ), and 2.9-3.6 kbar (average 3.5 $\pm 0.2 \mathrm{kbar}(1 \sigma)$ ).

Plagioclase in both samples has essentially identical and very limited compositional ranges of An19.4-An26.6 and Or0.6-Or1.7 (Table 1).

\section{Whole rock geochemistry}

Whole-rock major and trace element compositions (Table 2) were determined by X-ray fluorescence (Philips PW 1480) and inductively coupled plasma-mass spectrometry (ICP-MS) (PE 6100 DRC) at the Zanjan University geochemical laboratory. The REE were normalized to the chondrite values of Sun and McDonough (1989). For the spider diagram the trace element abundances were normalized to the primitive mantle values of Taylor and McLennan (1985). 
Table 1 Mineral compositions of quartz monzonite (G1) and granite (G2) of Poshteh Pluton

\begin{tabular}{|c|c|c|c|c|c|c|c|c|c|}
\hline \multirow[b]{3}{*}{$\mathrm{SiO}_{2}$} & \multicolumn{9}{|c|}{ Biotite } \\
\hline & \multirow[b]{2}{*}{41.6} & \multicolumn{4}{|c|}{ TR-103 (G2) } & \multirow[b]{2}{*}{40.64} & \multirow[b]{2}{*}{42.21} & \multicolumn{2}{|c|}{ TR-117 (G1) } \\
\hline & & 40.14 & 42.44 & 43.05 & 42.22 & & & 43.03 & 42.03 \\
\hline $\mathrm{TiO}_{2}$ & 2.25 & 2.62 & 2.59 & 2.26 & 2.76 & 2.98 & 2.66 & 3.6 & 3.55 \\
\hline $\mathrm{Al}_{2} \mathrm{O}_{3}$ & 10.37 & 11.17 & 9.65 & 8.93 & 10.81 & 11.66 & 12.16 & 10.69 & 11.31 \\
\hline $\mathrm{FeO}$ & 28.72 & 26.92 & 27.37 & 26.85 & 25.06 & 26.06 & 26.13 & 26.07 & 25.08 \\
\hline $\mathrm{LiO}$ & 0.21 & 0.19 & 0.19 & 0.31 & 0.25 & 0.26 & 0.12 & 0.05 & 0.08 \\
\hline $\mathrm{MnO}$ & 0.7 & 0.73 & 0.73 & 0.74 & 0.71 & 0.72 & 0.02 & 0.23 & 0.17 \\
\hline $\mathrm{MgO}$ & 5.2 & 4.98 & 4.02 & 4.95 & 6.5 & 4.24 & 4.61 & 5.01 & 6.21 \\
\hline $\mathrm{CaO}$ & 0.06 & $\mathrm{BD}<$ & $\mathrm{BD}<$ & $\mathrm{BD}<$ & 0.35 & 0.45 & 0.57 & 0.15 & 0.22 \\
\hline $\mathrm{Na}_{2} \mathrm{O}$ & 0.28 & 0.09 & 0.06 & 0.14 & 0.56 & 0.56 & 0.12 & 2.6 & 0.14 \\
\hline $\mathrm{K}_{2} \mathrm{O}$ & 9.15 & 10.71 & 10.17 & 10.3 & 8.14 & 10.12 & 10.21 & 9.19 & 9.31 \\
\hline $\mathrm{SrO}$ & $\mathrm{BD}<$ & $\mathrm{BD}<$ & $\mathrm{BD}<$ & $\mathrm{BD}<$ & $\mathrm{BD}<$ & $\mathrm{BD}<$ & $\mathrm{BD}<$ & $\mathrm{BD}<$ & $\mathrm{BD}<$ \\
\hline $\mathrm{Cr}_{2} \mathrm{O}_{3}$ & 0.01 & 0.03 & $\mathrm{BD}<$ & $\mathrm{BD}<$ & 0.01 & 0.01 & 0.01 & $\mathrm{BD}<$ & $\mathrm{BD}<$ \\
\hline $\mathrm{P}_{2} \mathrm{O}_{5}$ & 0.05 & $\mathrm{BD}<$ & $\mathrm{BD}<$ & 0.03 & 0.04 & 0.01 & 0.05 & 0.05 & 0.1 \\
\hline Total & 98.6 & 97.58 & 97.22 & 97.56 & 97.41 & 97.71 & 98.87 & 100.67 & 98.2 \\
\hline $\mathrm{Si}$ & 5.28 & 5.34 & 5.39 & 5.44 & 5.88 & 5.93 & 5.86 & 5.9 & 5.91 \\
\hline $\mathrm{Al}^{\mathrm{iv}}$ & 2.72 & 2.66 & 2.61 & 2.56 & 2.12 & 2.07 & 2.14 & 2.1 & 2.16 \\
\hline $\mathrm{Al}^{\mathrm{vi}}$ & 0.5 & 0.51 & 0.64 & 0.54 & 0.03 & 0.06 & 0.06 & 1.01 & 1.1 \\
\hline $\mathrm{Ti}$ & 0.27 & 0.31 & 0.3 & 0.26 & 0.31 & 0.37 & 0.34 & 0.29 & 0.22 \\
\hline $\mathrm{Cr}$ & $\mathrm{BD}<$ & $\mathrm{BD}<$ & $\mathrm{BD}<$ & $\mathrm{BD}<$ & $\mathrm{BD}<$ & $\mathrm{BD}<$ & $\mathrm{BD}<$ & $\mathrm{BD}<$ & $\mathrm{BD}<$ \\
\hline $\mathrm{Fe}$ & 3.77 & 3.52 & 3.58 & 3.49 & 3.01 & 3.51 & 3.48 & 3.61 & 3.51 \\
\hline $\mathrm{Li}$ & 0.08 & 0.07 & 0.07 & 0.1 & 0.09 & 0.09 & 0.04 & 0.01 & 0.02 \\
\hline $\mathrm{Mn}$ & 0.09 & 0.1 & 0.1 & 0.1 & 0.11 & 0.09 & 0.1 & 0.1 & 0.08 \\
\hline $\mathrm{Mg}$ & 1.22 & 1.16 & 0.94 & 1.15 & 1.21 & 1.15 & 1.16 & 1.17 & 1.17 \\
\hline $\mathrm{Ca}$ & 0.01 & $\mathrm{BD}<$ & $\mathrm{BD}<$ & $\mathrm{BD}<$ & 0.64 & 0.66 & 0.67 & 0.7 & 0.7 \\
\hline $\mathrm{Na}$ & 0.09 & 0.03 & 0.02 & 0.04 & 0.08 & 0.87 & 0.96 & 0.95 & 0.95 \\
\hline $\mathrm{K}$ & 1.83 & 2.14 & 2.03 & 2.04 & 2.12 & 2.02 & 1.83 & 2.03 & 2.03 \\
\hline$Y$ total & 5.91 & 5.76 & 5.76 & 5.86 & 5.66 & 5.75 & 5.69 & 5.7 & 5.7 \\
\hline$X$ total & 1.93 & 2.17 & 2.05 & 2.08 & 2.75 & 2.54 & 2.66 & 2.68 & 2.68 \\
\hline $\mathrm{Al}$ total & 3.22 & 3.17 & 3.25 & 3.1 & 3.15 & 3.13 & 3.19 & 3.11 & 3.11 \\
\hline $\mathrm{Fe} / \mathrm{Fe}+\mathrm{Mg}$ & 0.76 & 0.75 & 0.79 & 0.75 & 0.73 & 0.77 & 0.79 & 0.81 & 0.81 \\
\hline $\mathrm{Mn} / \mathrm{Mn}+\mathrm{Fe}$ & 0.02 & 0.03 & 0.03 & 0.03 & 0.03 & 0.04 & 0.11 & 0.04 & 0.04 \\
\hline Total Al & 3.22 & 3.17 & 3.25 & 3.1 & 3.15 & 3.13 & 3.19 & 3.11 & 3.11 \\
\hline \multicolumn{10}{|l|}{ Plagioclase } \\
\hline & \multicolumn{4}{|c|}{ TR-103 (G2) } & \multicolumn{3}{|c|}{ TR-117 (G1) } & & \\
\hline $\mathrm{SiO}_{2}$ & 61.28 & 61.51 & 62.74 & 62.72 & 60.61 & 61.4 & 61.74 & 62.06 & \\
\hline $\mathrm{TiO}_{2}$ & 0.03 & 0.01 & $\mathrm{Bd}>$ & 0.01 & $\mathrm{Bd}>$ & $\mathrm{Bd}>$ & $\mathrm{Bd}>$ & $\mathrm{Bd}>$ & \\
\hline $\mathrm{Al}_{2} \mathrm{O}_{3}$ & 23.87 & 23.36 & 23.7 & 23.6 & 24.69 & 23.48 & 23.76 & 23.87 & \\
\hline $\mathrm{FeO}$ & 0.07 & 0.1 & 0.03 & 0.01 & 0.07 & 0.02 & 0.11 & 0.06 & \\
\hline $\mathrm{CaO}$ & 6.03 & 5.95 & 4.64 & 4.4 & 5.71 & 5.86 & 5.82 & 5.44 & \\
\hline $\mathrm{Na}_{2} \mathrm{O}$ & 9.29 & 9.38 & 9.51 & 9.99 & 8.73 & 9.27 & 9.02 & 8.96 & \\
\hline $\mathrm{K}_{2} \mathrm{O}$ & 0.18 & 0.19 & 0.11 & 0.13 & 0.31 & 0.13 & 0.24 & 0.26 & \\
\hline $\mathrm{MgO}$ & $\mathrm{Bd}>$ & $\mathrm{Bd}>$ & 0.02 & $\mathrm{Bd}>$ & $\mathrm{Bd}>$ & 0.01 & $\mathrm{Bd}>$ & 0.03 & \\
\hline $\mathrm{MnO}$ & 0.01 & $\mathrm{Bd}>$ & 0.02 & $\mathrm{Bd}>$ & $\mathrm{Bd}>$ & 0.01 & $\mathrm{Bd}>$ & $\mathrm{Bd}>$ & \\
\hline $\mathrm{SrO}$ & $\mathrm{Bd}>$ & $\mathrm{Bd}>$ & $\mathrm{Bd}>$ & $\mathrm{Bd}>$ & $\mathrm{Bd}>$ & $\mathrm{Bd}>$ & $\mathrm{Bd}>$ & $\mathrm{Bd}>$ & \\
\hline $\mathrm{Cr}_{2} \mathrm{O}_{2}$ & $\mathrm{Bd}>$ & $\mathrm{Bd}>$ & $\mathrm{Bd}>$ & $\mathrm{Bd}>$ & $\mathrm{Bd}>$ & 0.03 & 0.02 & $\mathrm{Bd}>$ & \\
\hline $\mathrm{P}_{2} \mathrm{O}_{2}$ & 0.04 & $\mathrm{Bd}>$ & 0.02 & $\mathrm{Bd}>$ & 0.02 & $\mathrm{Bd}>$ & $\mathrm{Bd}>$ & 0.09 & \\
\hline Total & 100.8 & 100.5 & 100.79 & 100.86 & 100.14 & 100.21 & 100.71 & 100.77 & \\
\hline
\end{tabular}


Table 1 (continued)

\begin{tabular}{|c|c|c|c|c|c|c|c|c|}
\hline \multicolumn{9}{|c|}{ Plagioclase } \\
\hline \multirow[b]{2}{*}{$\mathrm{Si}$} & \multicolumn{4}{|c|}{ TR-103 (G2) } & \multicolumn{4}{|c|}{ TR-117 (G1) } \\
\hline & 10.87 & 10.94 & 11.05 & 11.05 & 10.79 & 10.94 & 10.93 & 10.97 \\
\hline $\mathrm{Ti}$ & $\mathrm{Bd}>$ & $\mathrm{Bd}>$ & $\mathrm{Bd}>$ & $\mathrm{Bd}>$ & $\mathrm{Bd}>$ & $\mathrm{Bd}>$ & $\mathrm{Bd}>$ & $\mathrm{Bd}>$ \\
\hline $\mathrm{Al}$ & 4.99 & 4.89 & 4.92 & 4.9 & 5.18 & 4.93 & 4.96 & 4.97 \\
\hline $\mathrm{Fe}(\mathrm{ii})$ & 0.01 & 0.01 & $\mathrm{Bd}>$ & $\mathrm{Bd}>$ & 0.01 & $\mathrm{Bd}>$ & 0.02 & 0.01 \\
\hline $\mathrm{Ca}$ & 1.15 & 1.13 & 0.88 & 0.83 & 1.09 & 1.12 & 1.1 & 1.03 \\
\hline $\mathrm{Na}$ & 3.19 & 3.23 & 3.25 & 3.41 & 3.01 & 3.2 & 3.1 & 3.07 \\
\hline $\mathrm{K}$ & 0.04 & 0.04 & 0.02 & 0.03 & 0.07 & 0.03 & 0.05 & 0.06 \\
\hline Total & 20.25 & 20.31 & 20.12 & 20.22 & 20.16 & 20.22 & 20.16 & 20.11 \\
\hline An & 26.15 & 25.7 & 21.11 & 19.44 & 26.1 & 25.71 & 25.95 & 24.77 \\
\hline $\mathrm{Ab}$ & 72.92 & 73.32 & 78.29 & 79.88 & 72.21 & 73.61 & 72.78 & 73.82 \\
\hline Or & 0.93 & 0.98 & 0.6 & 0.68 & 1.69 & 0.68 & 1.27 & 1.41 \\
\hline
\end{tabular}

Microprobe analyses

Biotite: $\mathrm{XFe}=100(\mathrm{Fe} 2++\mathrm{viFe} 3+) /(\mathrm{Mg}+\mathrm{Fe} 2++\mathrm{viFe} 3+) ; \mathrm{FeOt}=\mathrm{FeO}$ total

Plagioclase: $A b$ albite, $A n$ anorthite, $O r$ orthoclase

Fig. 5 a Classification diagrams for biotite in quartz monzonite $\mathrm{G} 1$ and granite $\mathrm{G} 2$ of the Poshteh Pluton (after Deer et al. 1992). b mica composition in coordinates $\mathrm{Mg}-\mathrm{Li}$ versus $\mathrm{Fe}+\mathrm{Mn}+\mathrm{Ti}-\mathrm{Al}$ (after Tischendorf et al. 1997)
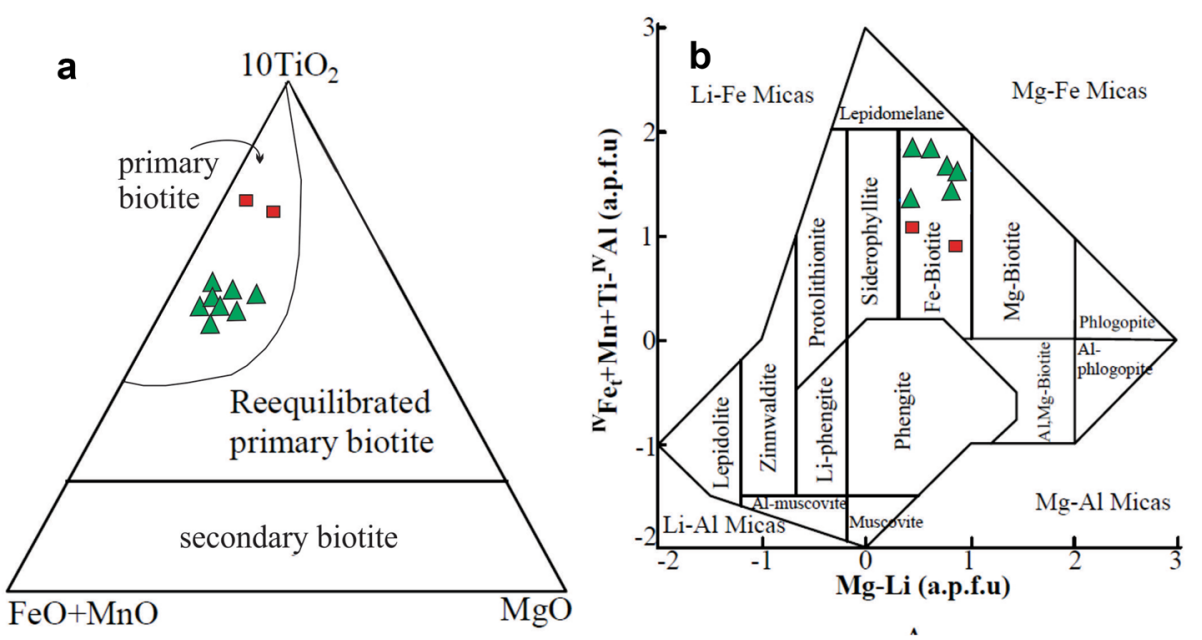

Posteh Pluton: $\quad$ G1 - quartz monzonite $\Delta \mathrm{G} 2$ : granite
The G1 quartz monzonite contains between 51 and $60 \%$ $\mathrm{SiO}_{2}, 11-13 \% \mathrm{Al}_{2} \mathrm{O}_{3}$ and $4-6 \% \mathrm{~K}_{2} \mathrm{O}+\mathrm{Na}_{2} \mathrm{O}$. The unit is metaluminous, high-K calc-alkaline (Fig. 6) and magnesian. Its chondrite normalized REE pattern is fractionated with enrichment of the LREE and almost no Eu anomalies (Fig. 7a). In the spider diagram the primitive mantle normalized pattern is quite uniform except for distinct negative anomalies for $\mathrm{Ba}, \mathrm{Nb}$ and $\mathrm{Ta}$ and positive anomalies for $\mathrm{Pb}$ (Fig. 7b).

The younger $\mathrm{G} 2$ granite has $69-76 \% \mathrm{SiO}_{2}, 10-13 \% \mathrm{Al}_{2} \mathrm{O}_{3}$ and $5-10 \% \mathrm{~K}_{2} \mathrm{O}+\mathrm{Na}_{2} \mathrm{O}$. The rocks are ferroan, high-K calcalkaline, and plot at the transition from metaluminous to peraluminous (Fig. 6). The REE pattern is parallel to that of G1, but at lower abundances and with a pronounced negative Eu anomaly (Fig. 7a). The data pattern in the spider diagram is also similar to that of G1, but it has lower abundances of all elements and stronger negative anomalies for $\mathrm{Ba}, \mathrm{Sr}$ and Eu (Fig. 7b).

\section{Geochronology}

Zircon were separated from two samples by crushing, pulverizing and various enrichment stages. Before analysis the grains were chemically abraded following Mattinson (2005). The data were obtained by ID-TIMS U-Pb geochronology in Oslo, following the procedure of Krogh (1973), with details documented in Corfu (2004). Decay constants are those of Jaffey et al. (1971). Plotting was done with the program Isoplot (Ludwig 2009). 
Table 2 Geochemical composition of of quartz monzonite (G1) and granite (G2) of Poshteh Pluton

\begin{tabular}{|c|c|c|c|c|c|}
\hline No & $\begin{array}{l}\mathrm{G}_{1} \\
\text { TR-107 }\end{array}$ & $\begin{array}{l}\mathrm{G}_{1} \\
\text { TR-115 }\end{array}$ & $\begin{array}{l}\mathrm{G}_{1} \\
\mathrm{TR}-117\end{array}$ & $\begin{array}{l}G_{2} \\
\text { TR-103 }\end{array}$ & $\begin{array}{l}G_{2} \\
\text { TR-105 }\end{array}$ \\
\hline \multicolumn{6}{|l|}{$[\%]$} \\
\hline $\mathrm{SiO}_{2}$ & 52.89 & 55.56 & 58.56 & 74.51 & 75.00 \\
\hline $\mathrm{TiO}_{2}$ & 1.18 & 0.76 & 0.20 & 0.09 & 0.11 \\
\hline $\mathrm{Al}_{2} \mathrm{O}_{3}$ & 16.62 & 16.52 & 11.55 & 12.97 & 12.96 \\
\hline $\mathrm{Fe}_{2} \mathrm{O}_{3}$ & 10.05 & 7.34 & 2.69 & 1.15 & 1.47 \\
\hline $\mathrm{BaO}$ & 0.08 & 0.07 & 0.33 & 0.05 & 0.07 \\
\hline $\mathrm{CaO}$ & 6.41 & 7.11 & 11.50 & 1.10 & 0.79 \\
\hline $\mathrm{K}_{2} \mathrm{O}$ & 2.50 & 1.88 & 3.03 & 4.40 & 4.79 \\
\hline $\mathrm{MgO}$ & 4.26 & 4.41 & 0.51 & 0.15 & 0.24 \\
\hline $\mathrm{MnO}$ & 0.21 & 0.16 & 0.08 & 0.03 & 0.20 \\
\hline $\mathrm{Na}_{2} \mathrm{O}$ & 3.61 & 3.16 & 3.08 & 4.03 & 3.70 \\
\hline $\mathrm{P}_{2} \mathrm{O}_{5}$ & 0.21 & 0.16 & 0.06 & 0.03 & 0.03 \\
\hline L.O.I & 1.94 & 2.86 & 8.42 & 1.44 & 0.81 \\
\hline SUM & 99.96 & 99.99 & 100.01 & 99.95 & 99.99 \\
\hline \multicolumn{6}{|l|}{ [ppm] } \\
\hline $\mathrm{Ag}$ & $<0.1$ & $<0.1$ & $<0.1$ & $<0.1$ & $<0.1$ \\
\hline $\mathrm{Al}$ & 80,812 & 82,940 & 58,678 & 65,153 & 63,286 \\
\hline As & 3.9 & 3.1 & 2.3 & 0.9 & 1.4 \\
\hline $\mathrm{Ba}$ & 574 & 498 & 2293 & 384 & 503 \\
\hline $\mathrm{Be}$ & 1.2 & 0.9 & 0.7 & 1.6 & 1.4 \\
\hline $\mathrm{Bi}$ & 0.3 & 0.3 & 0.2 & 0.2 & 0.2 \\
\hline $\mathrm{Ca}$ & 42,324 & 46,915 & 72,047 & 758 & 5181 \\
\hline $\mathrm{Cd}$ & $<0.1$ & $<0.1$ & $<0.1$ & $<0.1$ & $<0.1$ \\
\hline $\mathrm{Ce}$ & 22 & 21 & 20 & 40 & 47 \\
\hline $\mathrm{Co}$ & 18.9 & 16.6 & 3.6 & 2.4 & 2.1 \\
\hline $\mathrm{Cr}$ & 10 & 17 & 20 & 9 & 10 \\
\hline Cs & 1.9 & 1.8 & 1.2 & 1.9 & 2 \\
\hline $\mathrm{Cu}$ & 20 & 16 & 6 & 9 & 9 \\
\hline Dy & 3.78 & 2.53 & 0.8 & 1.83 & 1.48 \\
\hline Er & 2.07 & 1.46 & 1.31 & 0.86 & 0.66 \\
\hline $\mathrm{Eu}$ & 1.1 & 0.8 & 1.8 & 0.2 & 0.4 \\
\hline $\mathrm{Fe}$ & 56,974 & 43,624 & 17,739 & 7769 & 10,372 \\
\hline $\mathrm{Gd}$ & 1.6 & 1.19 & 1.16 & 1.74 & 1.65 \\
\hline In & $<0.5$ & $<0.5$ & $<0.5$ & $<0.5$ & $<0.5$ \\
\hline $\mathrm{K}$ & 15,410 & 14,620 & 19,091 & 27,769 & 26,487 \\
\hline $\mathrm{La}$ & 13 & 10 & 14 & 22 & 27 \\
\hline $\mathrm{Li}$ & 28 & 40 & 20 & 2 & 6 \\
\hline $\mathrm{Lu}$ & 0.31 & 0.2 & $<0.1$ & 0.14 & 0.12 \\
\hline $\mathrm{Mg}$ & 19,243 & $>2 \%$ & 3137 & 1164 & 1756 \\
\hline $\mathrm{Mn}$ & 1310 & 1090 & 516 & 213 & 186 \\
\hline Mo & 4.6 & $<0.1$ & 0.1 & $<0.1$ & $<0.1$ \\
\hline $\mathrm{Na}$ & 23,453 & 20,824 & 20,098 & 24,886 & 23,196 \\
\hline $\mathrm{Nb}$ & 7.5 & 4.7 & 3.4 & 7.3 & 6.8 \\
\hline $\mathrm{Nd}$ & 13 & 15.5 & 14.3 & 12.5 & 11.4 \\
\hline $\mathrm{Ni}$ & 5 & 5 & 4 & 2 & $<1$ \\
\hline $\mathrm{P}$ & 917 & 703 & 283 & 137 & 117 \\
\hline $\mathrm{Pb}$ & 9 & 2 & 4 & 7 & 9 \\
\hline $\operatorname{Pr}$ & 1.11 & 1.78 & 1.93 & 3.61 & 4.09 \\
\hline $\mathrm{Rb}$ & 78 & 80 & 41 & 147 & 123 \\
\hline S & 235 & 63 & 57 & 81 & 51 \\
\hline
\end{tabular}


Table 2 (continued)

\begin{tabular}{|c|c|c|c|c|c|}
\hline No & $\begin{array}{l}\mathrm{G}_{1} \\
\text { TR-107 }\end{array}$ & $\begin{array}{l}\mathrm{G}_{1} \\
\text { TR-115 }\end{array}$ & $\begin{array}{l}\mathrm{G}_{1} \\
\text { TR-117 }\end{array}$ & $\begin{array}{l}G_{2} \\
\text { TR-103 }\end{array}$ & $\begin{array}{l}G_{2} \\
\text { TR-105 }\end{array}$ \\
\hline $\mathrm{Sb}$ & 1 & 1.2 & $<0.5$ & 0.7 & $<0.5$ \\
\hline $\mathrm{Sc}$ & 12.7 & 14.6 & 2.4 & 2.1 & 2 \\
\hline $\mathrm{Se}$ & 5.38 & 18.37 & 26.12 & 11.39 & 5.31 \\
\hline $\mathrm{Sm}$ & 3.37 & 3.05 & 3.17 & 2.58 & 2.69 \\
\hline $\mathrm{Sn}$ & 2.3 & 1.8 & 1 & 2.1 & 1.7 \\
\hline $\mathrm{Sr}$ & 231 & 219 & 247 & 49 & 60 \\
\hline $\mathrm{Ta}$ & 0.59 & 0.33 & 0.14 & 0.63 & 0.44 \\
\hline $\mathrm{Tb}$ & 0.37 & 0.31 & 0.32 & 0.47 & 0.33 \\
\hline $\mathrm{Te}$ & 0.19 & 0.25 & 0.26 & $<0.1$ & $<0.1$ \\
\hline Th & 4.3 & 2.9 & 5.77 & 14.38 & 18.52 \\
\hline $\mathrm{Ti}$ & 6814 & 4505 & 1092 & 435 & 492 \\
\hline $\mathrm{Tl}$ & 0.31 & 0.25 & 0.17 & 0.46 & 0.51 \\
\hline $\mathrm{Tm}$ & 0.36 & 0.24 & $<0.1$ & 0.17 & 0.14 \\
\hline $\mathrm{U}$ & 0.66 & 0.5 & 0.4 & 1.7 & 2.3 \\
\hline $\mathrm{V}$ & 156 & 155 & 19 & 14 & 12 \\
\hline W & $<1$ & $<1$ & $<1$ & $<1$ & $<1$ \\
\hline $\mathrm{Y}$ & 20 & 12.9 & 3.2 & 9.3 & 6.8 \\
\hline $\mathrm{Yb}$ & 3.3 & 1.8 & 2.5 & 0.6 & 0.9 \\
\hline $\mathrm{Zn}$ & 102 & 174 & 29 & 10 & 15 \\
\hline \multirow[t]{2}{*}{ Coord } & $34^{\circ} 43^{\prime} 39^{\prime \prime} \mathrm{N}$ & $34^{\circ} 43^{\prime} 23^{\prime \prime} \mathrm{N}$ & $34^{\circ} 43^{\prime} 25^{\prime \prime} \mathrm{N}$ & $34^{\circ} 43^{\prime} 26^{\prime \prime} \mathrm{N}$ & $34^{\circ} 43^{\prime} 25^{\prime \prime} \mathrm{N}$ \\
\hline & $60^{\circ} 19^{\prime} 59^{\prime \prime} \mathrm{E}$ & $60^{\circ} 20^{\prime} 09^{\prime \prime} \mathrm{E}$ & $60^{\circ} 20^{\prime} 15^{\prime \prime} \mathrm{E}$ & $60^{\circ} 19^{\prime} 42^{\prime \prime} \mathrm{E}$ & $60^{\circ} 19^{\prime} 44^{\prime \prime} \mathrm{E}$ \\
\hline
\end{tabular}
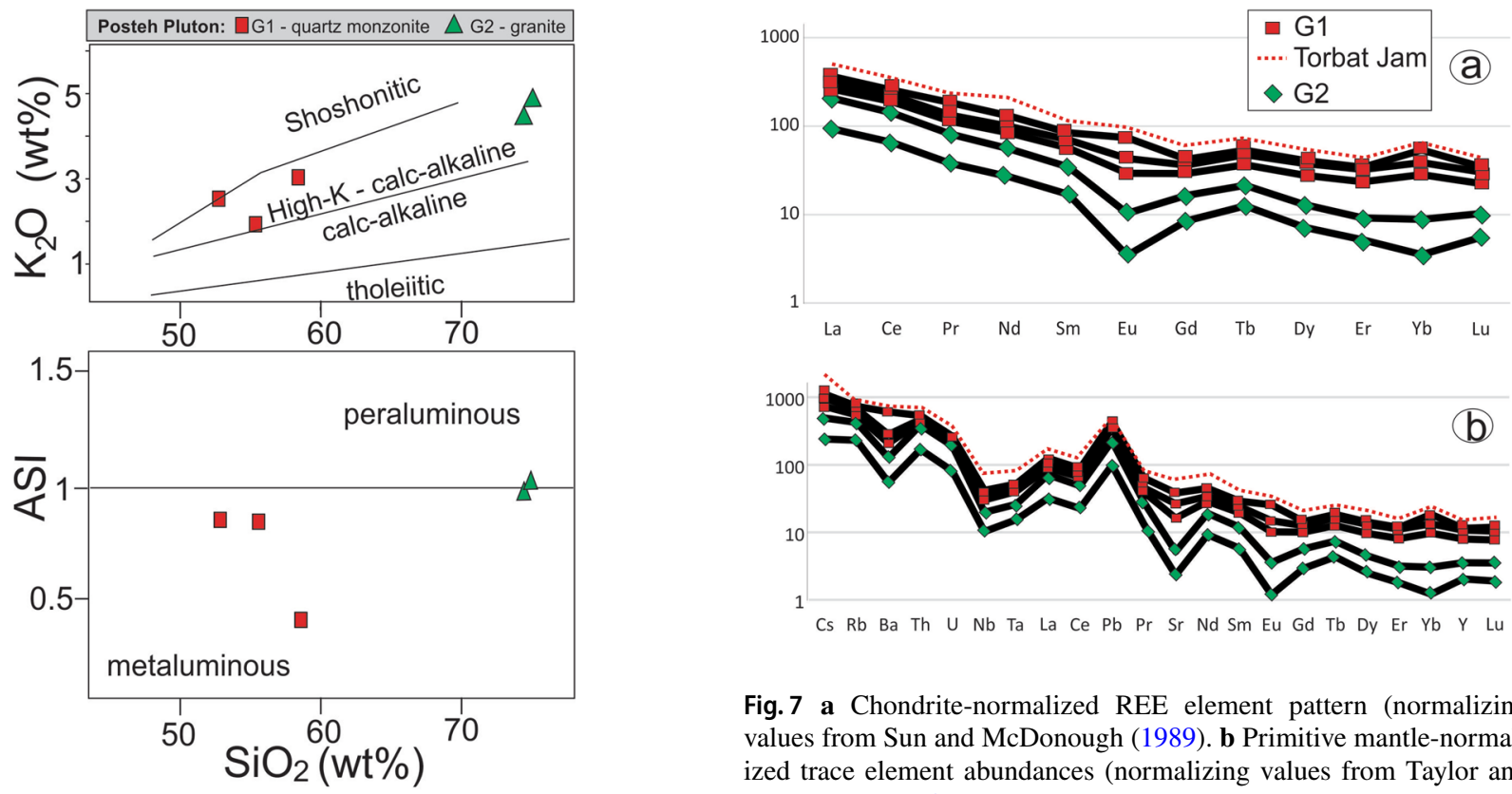

Fig. 7 a Chondrite-normalized REE element pattern (normalizing values from Sun and McDonough (1989). b Primitive mantle-normalized trace element abundances (normalizing values from Taylor and McLennan (1985)

Fig. 6 Above: $\mathrm{K}_{2} \mathrm{O}$ vs. $\mathrm{SiO}_{2}$ diagram (Peccerillo and Taylor 1976). Below: aluminum-saturation index ASI vs. $\mathrm{SiO}_{2}$ diagram (Shand 1947)

Sample SHR-104 represents the older quartz monzonite (G1) instrusion of the Poshteh Pluton. The zircon population consists largely of long prismatic crystals. Of the five analyses carried out (Table 3, Fig. 8a) two are concordant and two have some small amounts of older inherited $\mathrm{Pb}$, 


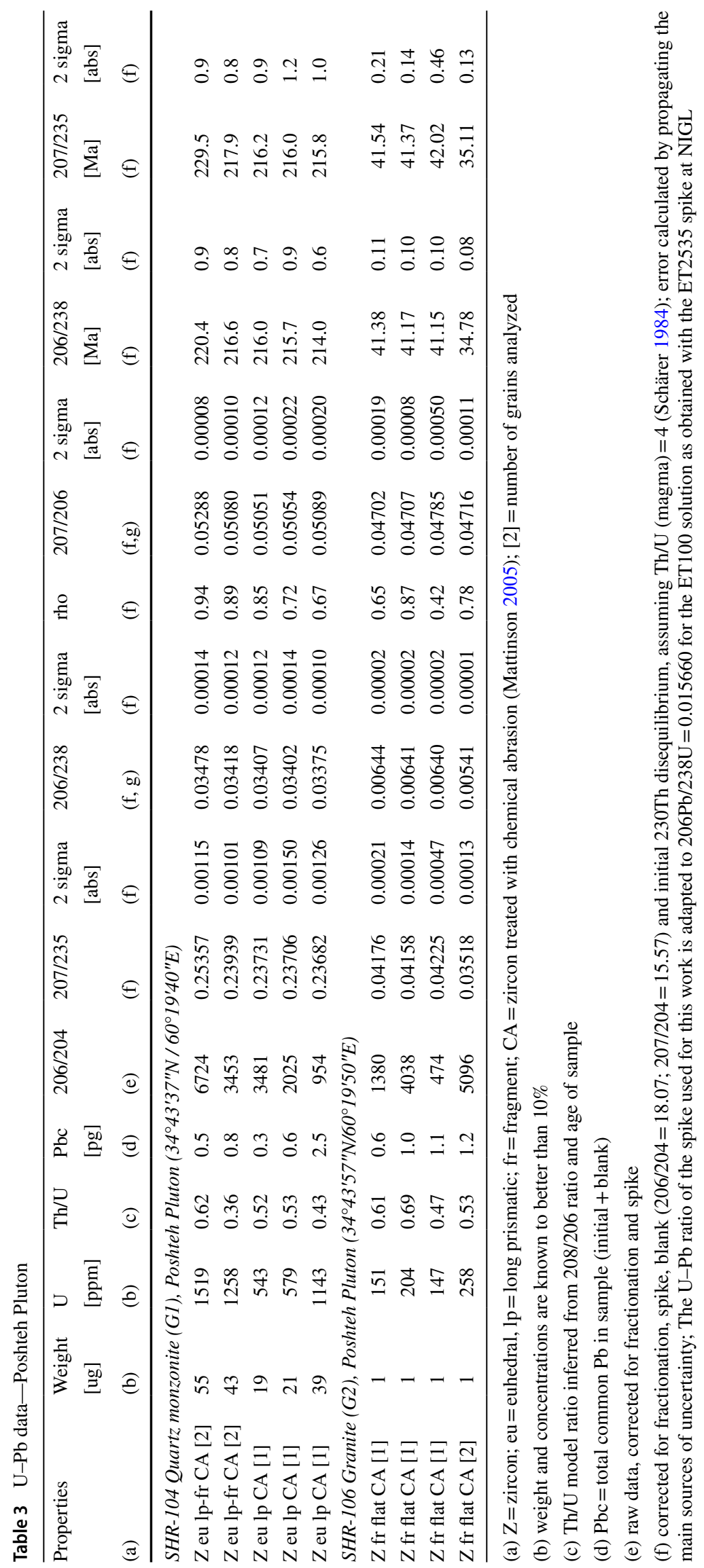



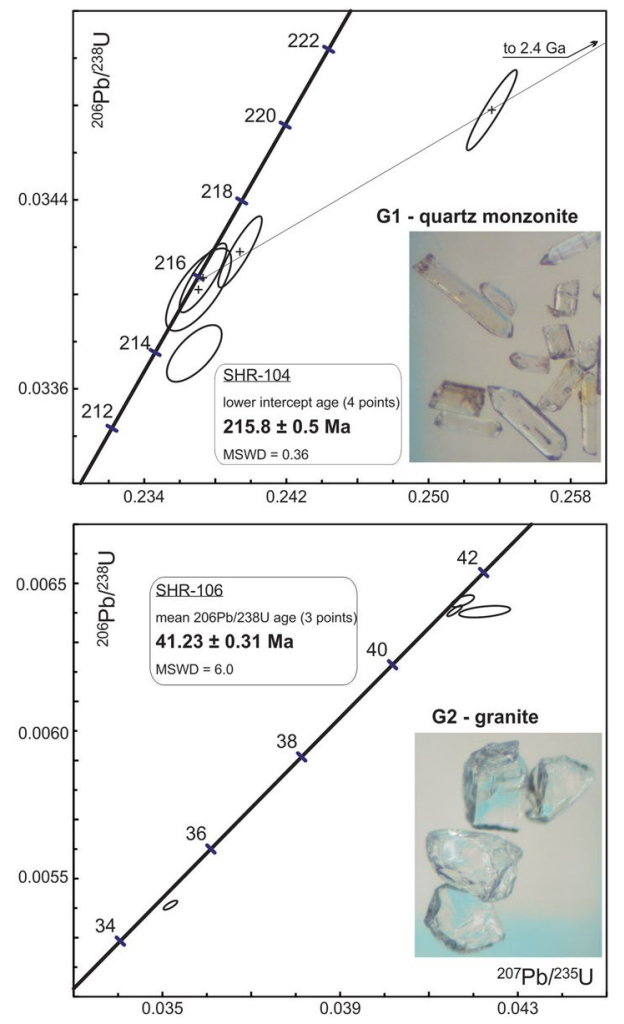

Fig. 8 Concordia diagrams with $\mathrm{U}-\mathrm{Pb}$ data for zircon in samples representing the two intrusive units G1 and G2 of the Poshteh Pluton. Ellipses indicate the 2 sigma uncertainty

together defining a discordia line with a lower intercept age of $215.8 \pm 0.5 \mathrm{Ma}(\mathrm{MSWD}=0.36)$. The line projects towards an upper intercept age of $2.4 \mathrm{Ga}$. A fifth data point plots below the line, probably because of some $\mathrm{Pb}$ loss.

Sample SHR-106 is a granite representing G2. The extracted zircon grains were mostly fragments of crystals, with some euhedral faces. Three of the analyses are clustered near the Concordia curve and yield an average ${ }^{206} \mathrm{~Pb} /{ }^{238} \mathrm{U}$ age of $41.23 \pm 0.31 \mathrm{Ma}$ (Fig. $8 \mathrm{~b}$ ). There is some scatter (MSWD $=6.0$ ), which, however, is propagated into the proportionally large uncertainty. A fourth analysis provides a much younger apparent age, which is ascribed to $\mathrm{Pb}$ loss.

\section{Discussion}

\section{Quartz monzonite (G1) and the Eo-Cimmerian Orogeny}

The Paleotethys opened in the Paleozoic, diachronously from west to east (e.g. Bagheri and Stampfli 2008; Stampfli et al. 2013; Torsvik and Cocks 2013; Jamei et al. 2020). Northward subduction underneath the Turan Plate (Eurasia) consumed the Paleotethys Ocean in the Carboniferous and Permian. This process is recorded by arc sequences, by ophiolites and by sedimentary rocks along, and north of the Paleotethys suture zone in northern Iran, and further east in Afghanistan and central Asia (Alavi 1991; Natal'in and Sengör 2005; Zanchetta et al. 2013; Shafaii Moghadam et al. 2015a; Siehl 2017).

Closure of this ocean and collision of the Central Iranian terranes with the Eurasian margin occurred during the Eo-Cimmerian Orogeny. In the Aghdarband Basin (Fig. 2) continental sediments of the late Triassic Miankuhi Formation, which lie unconformably on late Triassic marine shales (top of Sina Formation), were the youngest ones affected by this event (Mazaheri-Johari et al. 2021, 2022). Rocks and structures formed during this orogenic phase are cut by late tectonic plutons such as the 217-200 Ma Mashhad granitoids (Karimpour et al. 2010; Mirnejad et al. 2013; Deyhimi et al. 2019) and the coeval Torbat Jam granite about $100 \mathrm{~km}$ further to the SE (Fig. 2; Zanchetta et al. 2013). These plutons postdate major deformation and thrusting and are, thus, clearly late-tectonic. The deformed orogen was then covered by the Shemshak Group, a regionally widespread molasse-type deposit formed in the concluding stages of the Eo-Cimmerian Orogeny, in the latest Triassic and early Jurassic (Zanchi et al. 2009a; Wilmsen et al. 2009).

The G1 quartz monzonite of the Poshteh Pluton, dated here at $215.8 \pm 0.5 \mathrm{Ma}$, is thus coeval with the Mashhad and Torbat Jam plutons, suggesting that it has a similar tectonic origin related to the Eo-Cimmerian collision. There are also geochemical similarities between these units. The REE pattern of the Torbat Jam granodiorite (Zanchetta et al. 2013; Ghavi et al. 2018) is parallel to that of G1, with just slightly higher abundances, and the same similarity is evident for the PM-normalized trace elements (Fig. 7a, b). The REE patterns reported by Karimpour et al. (2010) and Mirnejad et al. (2013) for various phases of the Mashhad plutons (not plotted) are also similar to those of the Poshteh quartz monzonite, with essentially no Eu anomalies and similar to slightly lower overall abundances. Mirnejad et al. (2013) distinguish an earlier phase of I type affinity related to subduction and a subsequent phase of S-type affinity related to crustal melting. Karimpour et al. (2010) concluded that the Mashhad granitoids are S-type plutons, also consistent with the presence of abundant xenocrystic zircons. Some xenocrystic zircon is also present in the Postheh quartz monzonite, reflecting the presence of crustal components, but the geochemistry does not conform to the S-type criteria.

The trace element plots in Fig. 9 also reveal some differences between the various Triassic plutons, implying slight diverging tectonic conditions. The plots of $\mathrm{Rb}$ vs. $\mathrm{Ta}+\mathrm{Yb}$ and $\mathrm{Ta}$ vs. $\mathrm{Yb}$ (Fig. 9a, c) place Poshteh firmly in the volcanic arc sector whereas several of the Mashhad and Torbat Jam intrusions have higher abundances of $\mathrm{Rb}$ and especially of $\mathrm{Ta}$, which place them close to, or inside the 

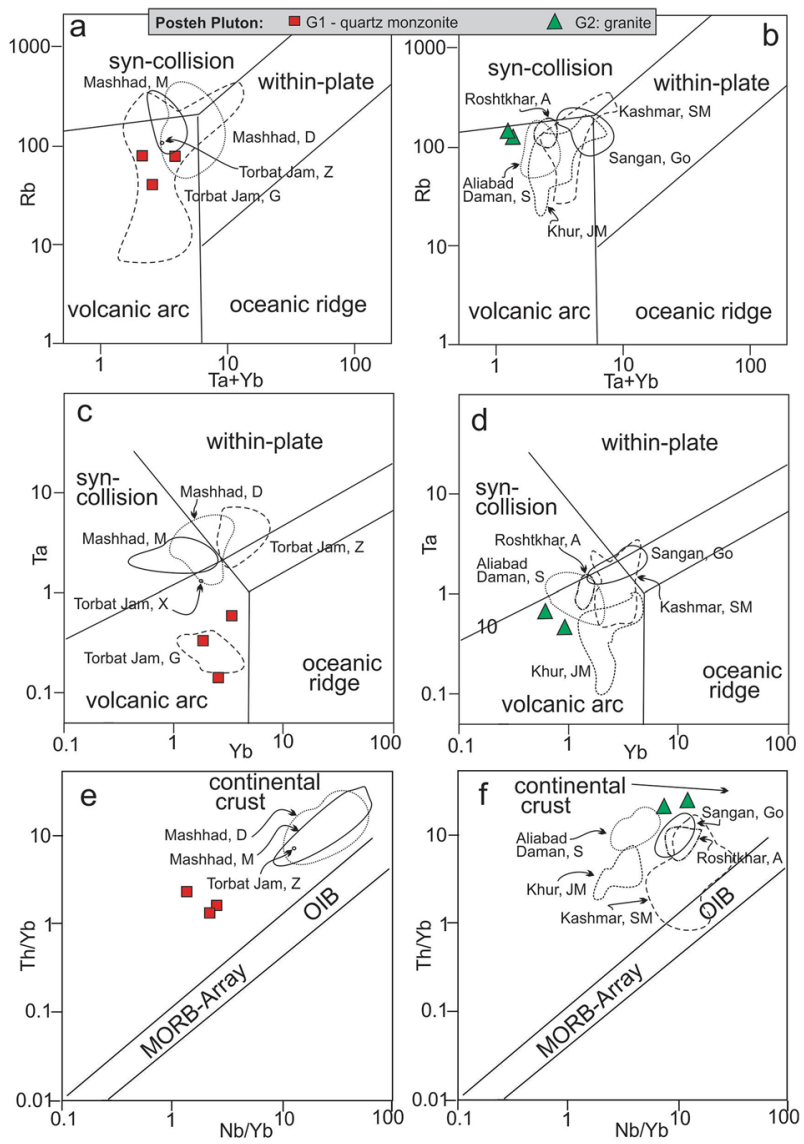

Fig. 9 Geochemical classification plots. a, c, e Triassic G2 quartz monzonite of the Poshteh Pluton and comparison with data for Torbat Jam and Mashhad plutons. b, d, f Eocene G1 granite of Poshteh Pluton and comparison with data for approximately coeval plutonic suites in southeastern Iran. Rb vs. $\mathrm{Yb}+\mathrm{Ta}$ and $\mathrm{Ta}$ vs. $\mathrm{Yb}$ plots (after Pearce et al. 1984); Th/Yb vs. Nb/Yb (after Pearce 2008). A=Alizadeh et al. (2018); $\mathrm{D}=$ Deyhimi et al. (2019); $\mathrm{G}=$ Ghavi et al. (2018); Go=Golmohammadi et al. (2015); JM=Javidi Moghaddam et al. (2020); $M=$ Mirnejad et al. (2013); $S=$ Shahbazi et al. (2021); $\mathrm{SM}=$ Shafaii Moghadam et al. (2015b); $\mathrm{Z}=$ Zanchetta et al. (2013)

syn-collisional field. The plot of $\mathrm{Th} / \mathrm{Yb}$ vs. $\mathrm{Nb} / \mathrm{Yb}$ (Fig. 9e) also shows a distinct deviation between Poshteh and the Mashhad and Torbat Jam intrusions, with a stronger affinity to continental crust of the latter. These trends likely reflect variations in geographical terms with respect to the tectonic suture, possibly combined with slight temporal trends related to the progress of the convergence and collision.

\section{The anomalous position of the Postheh Pluton south of the main Paleotethys suture}

An Eo-Cimmerian syn-collisional origin of the Poshteh G1 quartz-monzonite is thus supported by the timing and composition of this unit, and similarities with the plutons in the Mashhad-Torbat Jam area. There is, however, a major difference in the setting. The Mashhad-Torbat Jam granitoids

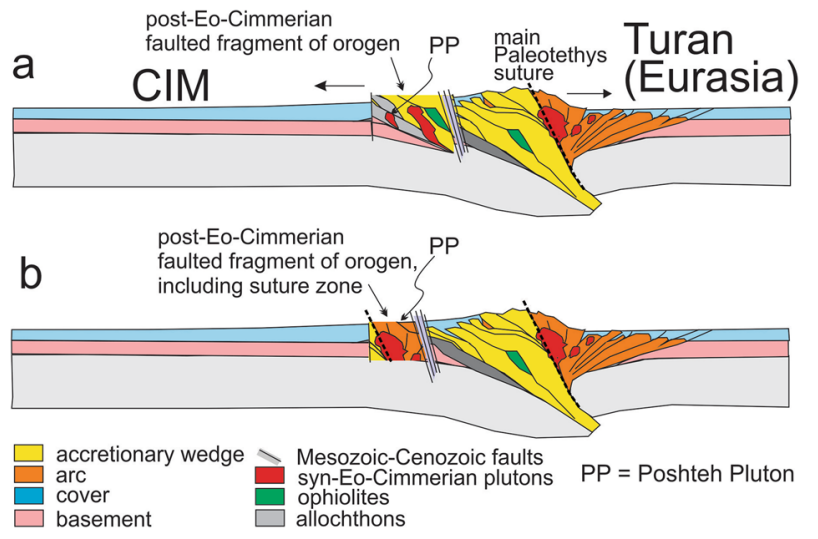

Fig. 10 Conceptual model ( modified from Zanchi et al. (2016)) with possible explanations for the position of the Triassic Poshteh quartz monzonite in the lower plate south of the main Eo-Cimmerian suture. Except for the suggested extension and faulting, the complexities that modified the area in the Mesozoic and Cenozoic are not considered. a The Postheh quartz monzonite is hosted in an allochthonous block detached from the orogen by Mesozoic-Cenozoic extension and faulting and moved to the present position. $\mathbf{b}$ The same general model, but in this case the fragment is from the core of the orogen and includes part of the suture

were emplaced in an assemblage of Devonian to Permian ophiolites, arc volcanic rocks and a major flysch accretionary complex, all located inside and north of the Paleotethys suture (Fig. 2). By contrast, the Postheh Pluton is located south of the Paleotethys suture where it intruded a sequence of supracrustal rocks presumed to be Proterozoic by Ternet et al. (1980) but still undated due to the lack of fossils.

A potential explanation could be that the analogy to the Mashhad-Torbat Jam rocks is just accidental, and that the Poshteh quartz monzonite developed in a different setting altogether, within the colliding plate but still as an expression of the same orogenic process.

The alternative, and probably more realistic, interpretation is that the Posteh quartz monzonite and surrounding rocks represent a tectonically disrupted allochthonous fragment of the Eo-Cimmerian Orogen (Fig. 10). This explanation has been proposed for the Anarak, Jandaq and Poshte-Badam metamorphic complexes, which are located in the northwestern part of the CIM south of the Doruneh Fault (Fig. 1; Bagheri and Stampfli 2008). Another example is the coeval late Triassic suite of tonalite-granite documented in the Saghand area on the western side of the CIM (Fig. 1; Ramezani and Tucker 2003). The Anarak Metamorphic Complex and associated units comprise a range of lithologies including metapelites, marbles, mafic volcanic rocks, locally with pillow lavas and ultramafic rocks. Units of the Anarak Metamorphic Complex preserve a record of Carboniferous and Permian magmatism and tectonism and locally high pressure metamorphism (Bagheri and Stampfli 2008; Zanchi et al. 2009a, 2015). Carboniferous orogenic activity 
has been documented from the Jandaq segment (Berra et al. 2017).

Based on paleomagnetic and geological data Davoudzadeh et al. (1981) deduced that the CIM had undergone a $135^{\circ}$ anticlockwise rotation since the Triassic. They suggested that the rotation could have sliced off a margin of the Turanian Plate and its orogenic cover and moved it to its present location at Nakhlak. Muttoni et al. (2009), however, expressed a note of caution on the magnitude of this rotation. More recent paleomagnetic studies (Mattei et al. 2012, 2015) confirm the occurrence of counterclockwise rotations in the CIM, both in the early Cretaceous and the Miocene, although of lesser magnitude than previously suggested. The position of the exotic Eo-Cimmerian crustal fragments has been interpreted by Bagheri and Stampfli (2008) as the consequence of Cretaceous extension in the Neotethys ocean system, which opened a back-arc basin, forming the Sabzevar and related ophiolites and detaching Eo-Cimmerian fragments from the margin of the Turan plate. The displacement was further enhanced by fragmentation and faulting between the various blocks of the CIM and considerable anti-clockwise rotation of the block reflecting the stress caused by the larger scale interaction between the Eurasian, Arabian and Indian plates. This events would have transferred fragments of the Paleotethys suture. Zanchi et al. (2021) expand this concept by linking the two stage counterclockwise rotation of the CIM (Mattei et al. 2012, 2015) and a proposed original identity of the Sabzevar and Nain ophiolites (Fig. 1; Pirnia et al. 2020). Accordingly, counterclockwise rotation of the CIM in the Jurassic-Cretaceous opened an oceanic system linked to the Sistan Ocean, forming a common Sabzevar-Nain ophiolite. The rotation also disrupted elements of the Paleotethys suture and carried them westward along the margin of the CIM. A second stage of counterclockwise rotation in the Cenozoic split the Nain from the Sabzevar ophiolite, moving it southwestward to its present position. and also transferred the Anarak and Nakhlak Paleotethys elements.

Alavi et al. (1997) proposed an alternative hypothesis whereby the exotic orogenic fragments such as Nakhlak were originally part of allochthonous nappes thrust onto the CIM plate, and subsequently further disrupted by plate rotations and faulting.

For the exotic Postheh occurrence dated in this study it is not possible to be certain on the preferred mechanism that was responsible for the present location south of the Paleotethys suture. The quartz monzonite and host rocks are entirely isolated in the expanse of post-Cretaceous rocks, which were themselves also extensively disrupted by faulting and thrusting (Fig. 2). The Alavi hypothesis, which combines Eo-Cimmerian nappe thrusting and later strike slip faulting, would seem to be the more plausible process. In its simplest terms, the alternative model of Zanchi et al.
(2021), which proposes the transport of Paleothesis suture fragments to their present locations piggy-back on slices of the CIM during two stages of post-Triassic anticlockwise rotation, would imply an origin of Postheh south of the Paropamisus belt (Fig. 1). This zone, representing the Paleotethys suture in Afghanistan, comprises a row of late Triassic plutons, continuing the trend set in the Mashhad region (Siehl 2017). The domains south of the Paropamisus are highly fragmented and faulted. The Doruneh Fault system widens eastward and appears to terminate at the important $\mathrm{N}-\mathrm{S}$ fault near the boundary to Afghanistan (Fig. 1). In the Ferdows thrust and fold system, west of Poshteh (Fig. 1), Mattei et al. (2020) document local clockwise rotation. This suggests that combinations of left- and right-lateral faulting in the eastern Doruneh Fault system may have controlled the translation of Poshteh but the potential links remain speculative. Detailed structural analyses of the eastern Doruneg Fault termination should provide key information on these processes.

\section{Granite (G2) and the Eocene magmatic stage}

Emplacement of the younger granite $\mathrm{G} 2$ at $41.23 \pm 0.31 \mathrm{Ma}$ coincides with a period of intense magmatism across the entire region, and especially in the Sistan suture zone (Camp and Griffis 1982; Shafaroudi et al. 2013; Golmohammadi et al. 2015; Mohammadi et al. 2016; Javidi Moghaddam et al. 2020; Shahbazi et al. 2021; Rezaei-Kahkhaei et al. 2021). There are geochemical similarities between the $G 2$ granite of the Poshteh Pluton and similar coeval intrusions such as at Sangan (Fig. 1), for example, the gently sloping REE pattern with distinct negative anomalies and negative anomalies of $\mathrm{Nb}$ and $\mathrm{Sr}$ (Fig. 7; Golmohammadi et al. 2015). In the plots of $\mathrm{Rb}$ vs. $\mathrm{Ta}+\mathrm{Yb}$ and Ta vs. $\mathrm{Yb}$ (Fig. 9b, d) the Poshteh granite corresponds broadly to the position of other coeval intrusions though the region, albeit somewhat displaced toward lower $\mathrm{Yb}$ and $\mathrm{Ta}+\mathrm{Yb}$ abundances, still in the volcanic arc field but already leaning towards a syncollisional character. The plot of $\mathrm{Th} / \mathrm{Yb}$ vs. $\mathrm{Nb} / \mathrm{Yb}$ (Fig. 9f) indicates a pronounced affinity with continental crust, similar but more extreme than the other comparable intrusions.

Golmohammadi et al. (2015) concluded that the chemical composition of the granitoids indicates an origin related to subduction. Zarrinkoub et al. (2012), however, pointed out that a subduction origin of this suite was not possible, because other evidence suggested closing of the Sistan Ocean already in the late Cretaceous. They proposed instead that this magmatic phase was related to orogenic collapse and detachment of thickened lithosphere, an analogous conclusion as reached by Pang et al. (2013), and by Rossetti et al. (2014) for central Iran. A process involving lithospheric delamination and asthenospheric upwelling causing widespread melting of the sub-continental lithospheric mantle is also favoured by Omidianfar et al. (2020). 
In the Poshteh Pluton the G2 intrusions were to some degree controlled by the northwest structure related to the Doruneh system. suggesting a close connection between regional deformation and magmatism. Bagheri and Gol (2020) related oroclinal buckling in the East Iranian Orogen to westward escape of the Afghan (Helmand) block as a consequence of the collision of India with Eurasia. These authors interpret the sequence of tectonic and magmatic events to result from lithospheric delamination, which caused the widespread regional Eocene to Miocene magmatic activity. The processes observed in the Poshteh Pluton can well be explained by this model.

\section{Conclusions}

The Poshteh area near Taybad, at the northeastern margin of the CIM, contains a fragment of the Eo-Cimmerian Orogen marked by late tectonic quartz monzonite (G1), which intruded at $215.8 \pm 0.5 \mathrm{Ma}$ during the collisional stage of the orogeny after closing of the Paleotethys. The quartz monzonite is geochemically similar to coeval plutons near Mashhad and Torbat Jam. The latter intruded Carboniferous-Permian arc sequences, ophiolites and flysch, which formed at the Paleotethys convergent margin. The Poshteh quartz monzonite (G1) is instead located south of the main Paleotethys suture. It may represent an Eo-Cimmerian element developed within the colliding upper plate, but then tectonically transferred to the lower plate, in a similar way as the occurrences farther west at Anarak and Saghand.

The younger G2 granite in the Poshteh Pluton formed at $41.23 \pm 0.31 \mathrm{Ma}$. Its emplacement was also accompanied by widespread hydrothermal alteration that developed locally important Fe mineralization in the country rocks. The geochemical composition of this granite is comparable to that of coeval and abundant late Eocene to Oligocene plutons in the region. It can be attributed to widespread melting of the subcontinental lithosphere related to delamination, presumably linked to the formation of the Eastern Iranian Orocline as suggested by Bagheri and Gol (2020).

Acknowledgements Gunborg Bye Fjeld performed the mineral separation. Incisive and constructive reviews by S. Bagheri, A. Zanchi and two anonymous reviewers are greatly appreciated.

Funding Open access funding provided by University of Oslo (incl Oslo University Hospital).

Open Access This article is licensed under a Creative Commons Attribution 4.0 International License, which permits use, sharing, adaptation, distribution and reproduction in any medium or format, as long as you give appropriate credit to the original author(s) and the source, provide a link to the Creative Commons licence, and indicate if changes were made. The images or other third party material in this article are included in the article's Creative Commons licence, unless indicated otherwise in a credit line to the material. If material is not included in the article's Creative Commons licence and your intended use is not permitted by statutory regulation or exceeds the permitted use, you will need to obtain permission directly from the copyright holder. To view a copy of this licence, visit http://creativecommons.org/licenses/by/4.0/.

\section{References}

Alavi M (1991) Sedimentary and structural characteristics of the Paleo-Tethys remnants in northeastern Iran. Geo Soc Am Bull 103:983-992

Alavi M, Vaziri H, Seyed Enami K, Lasemi Y (1997) The Triassic and associated rocks of the Nakhlak and Aghdarband areas in central and northeastern Iran as remnants of the southern Turanian active continental margin. Geol Soc Am Bull 109:1563-1575

Alavi-Naini M, Mossavi-Khorzughi E (2006) Geological map of Torbat-E-Jam. Geological Survey of Iran, sheet 8160, map scale $1 / 100000$

Alavi-Naini M, Ternet Y, Guillou Y, Maurizot P (1980) Geological map of Khaf, Geological Survey of Iran, sheet 8059, map scale $1 / 100000$

Alizadeh E, Ghadami G, Esmaeily D, Ma C, Lentz DR, Omrani J, Golmohammadi A (2018) Origin of $1.8 \mathrm{Ga}$ zircons in Post Eocene mafic dikes in the Roshtkhar area. NE Iran Intern Geol Rev 60:1855-1882. https://doi.org/10.1080/00206814.2017.13962

Bagheri S, Gol SD (2020) The Eastern Iranian Orocline. Earth-Sci Rev. https://doi.org/10.1016/j.earscirev.2020.103322

Bagheri S, Stampfli GM (2008) The Anarak, Jandaq and Posht-eBadam metamorphic complexes in central Iran: new geological data, relationships and tectonic implications. Tectonophys 451:123-155. https://doi.org/10.1016/j.tecto.2007.11.047

Berberian M, King GCP (1981) Towards a paleogeography and tectonic evolution of Iran. Can J Earth Sci 18:210-265. https://doi. org/10.1139/e81-163

Berra F, Zanchi A, Angiolini L, Vachard D, Vezzoli G, Zanchetta S, Bergomi M, Javadi HR, Kouhpeyma M (2017) The upper Palaeozoic Godar-e-Siah Complex of Jandaq: evidence and significance of a North Palaeotethyan succession in Central Iran. J Asian Earth Sci 138:272-290

Besse J, Torcq F, Gallet Y, Ricou LE, Krystyn L, Saidi A (1998) Late Permian to Late Triassic palaeomagnetic data from Iran: constraints on the migration of the Iranian block through the Tethyan Ocean and initial destruction of Pangaea. Geophys J Int 135:77-92

Camp VE, Griffis R (1982) Character, genesis and tectonic setting of igneous rocks in the Sistan suture zone, eastern Iran. Lithos $15: 221-239$

Corfu F (2004) U-Pb age, setting and tectonic significance of the Anorthosite-Mangerite-Charnockite-Granite suite, LofotenVesteralen, Norway. J Pet 45:1799-1819. https://doi.org/10.1093/ petrology/egh034

Davoudzadeh M, Soffel H, Schmidt K (1981) On the rotation of the Central East-Iran microplate. Neues Jb Geol Paläont Mh 1981-3:180-192

Deer WA, Howie RA, Zussman J (1992) An Introduction to the Rock Forming Minerals, 2nd edn. Longman, London, p 696

Deyhimi D, Kananian A, Mirnejad H, Sepidbar F, Vlastelic I, Paquette J-L, Barbarin B (2019) Zircon U-Pb geochronology, major-trace elements and $\mathrm{Sr}-\mathrm{Nd}$ isotope geochemistry of Mashhad granodiorites (NE Iran) and their mafic microgranular enclaves: evidence for magma mixing and mingling. Intern Geol Rev. https://doi.org/ $10.1080 / 00206814.2019 .1600435$ 
Domeier M (2018) Early Paleozoic tectonics of Asia: towards a fullplate model. Geosci Front 9:789-862. https://doi.org/10.1016/j. gsf.2017.11.012

Farbod Y, Bellier O, Shabanian E, Abbassi MR (2011) Geomorphic and structural variations along the Doruneh fault system (central Iran). Tectonics 30:TC6014. https://doi.org/10.1029/2011T C002889

Gaina C, van Hinsbergen DJJ, Spakman W (2015) Tectonic interactions between India and Arabia since the Jurassic reconstructed from marine geophysics, ophiolite geology, and seismic tomography. Tectonics. https://doi.org/10.1002/2014TC003780

Ghavi J, Karimpour MH, Mazaheri SA, Pan Y (2018) Triassic I-type granitoids from the Torbat e Jam area, northeastern Iran: petrogenesis and implications for Paleotethys tectonics. J Asian Earth Sci 164:159-178

Golmohammadi A, Karimpour MH, Shafaroudi AM, Mazaheri SA (2015) Alteration-mineralization, and radiometric ages of the source pluton at the Sangan iron skarn deposit, northeastern Iran. Ore Geol Rev 65:545-563

Golonka J (2004) Plate tectonic evolution of the southern margin of Eurasia in the Mesozoic and Cenozoic. Tectonophys 381:235-273

de Gramont XB, Guillou Y, Maurizot P, Vaslet D, de la Villeon H (1984) Geological map of Kariz Now. Geological Survey of Iran, sheet 8060, map scale $1 / 100000$

Henry DJ, Guidotti CV, Thomson JA (2005) The Ti-saturation surface for low-to medium pressure metapelitic biotites: implications for geothermometry and Ti substitution mechanisms. Am Mineral 90:316-328

Jaffey AH, Flynn KF, Glendenin LE, Bentley WC, Essling AM (1971) Precision measurement of half-lives and specific activities of ${ }^{235} \mathrm{U}$ and ${ }^{238}$ U. Phys Rev C 4:1889-1906. https://doi.org/10.1103/PhysR evC.4.1889

Jamei S, Ghorbani M, Williams IS, Moayyed M (2020) Tethyan oceans reconstructions with emphasis on the Early Carboniferous PirEshagh A-type rhyolite and the Late Palaeozoic magmatism in Iran. Inter Geol Rev. https://doi.org/10.1080/00206814.2020. 1768443

Javadi H, Ghassemi M, Shahpasandzadeh M, Guest B, Ashtiani M, Yassaghi A, Kouhpeyma M (2013) History of faulting on the Doruneh Fault System: implications for the kinematic changes of the Central Iranian Microplate. Geol Mag 150:651-672. https:// doi.org/10.1017/S0016756812000751

Javidi Moghaddam M, Karimpour MH, Shafaroudi AM, Santos JF, Corfu F (2020) Middle Eocene magmatism in the Khur region (Lut Block, Eastern Iran): implications for petrogenesis and tectonic setting. Int Geol Rev. https://doi.org/10.1080/00206814. 2019.1708815

Karimi S, Behzadi M, Rasa I, Ghazvinizadeh AM (2012) Geochemistry, fluid inclusion studies and origin of Ileh iron deposit, SW of Taibad (Khorasan Razavi). Petrol 33-12:1-20

Karimpour MH, Stern CR, Farmer L (2010) Zircon U-Pb geochronology, $\mathrm{Sr}-\mathrm{Nd}$ isotope analyses, and petrogenetic study of the Dehnow diorite and Kuhsangi granodiorite (Paleo-Tethys), NE Iran. J Asian Earth Sci 37:384-393. https://doi.org/10.1016/j. jseaes.2009.11.001

Krogh TE (1973) A low-contamination method for hydrothermal decomposition of zircon and extraction of $\mathrm{U}$ and $\mathrm{Pb}$ for isotopic age determinations. Geochim Cosmochim Acta 37:485-494. https://doi.org/10.1016/0016-7037(73)90213-5

Ludwig KR (2009) Isoplot 4.1. A geochronological toolkit for Microsoft Excel. Berkeley Geochron Centre Spec Publ 4:76

Mattei M, Cifelli F, Muttoni G, Zanchi A, Berra F, Mossavvari F, Eshraghi SA (2012) Neogene block-rotation in Central Iran: evidence from paleomagnetic data. Geol Soc Am Bull 124:943-956. https://doi.org/10.1130/B30479.1
Mattei M, Cifelli F, Muttoni G, Rashid H (2015) Post-Cimmerian (Jurassic-Cenozoic) paleogeography and vertical axis tectonic rotations of Central Iran and the Alborz Mountains. J Asian Earth Sci 102:92-101. https://doi.org/10.1016/j.jseaes.2014.09.038

Mattei M, Cifelli F, Alimohammadian H, Rashid H (2020) The role of active strike-slip faults and opposite vertical axis rotations in accommodating Eurasia-Arabia shortening in central Iran. Tectonophysics 774:228243. https://doi.org/10.1016/j.tecto.2019. 228243

Mattinson JM (2005) Zircon U-Pb chemical abrasion (“CA-TIMS”) method: combined annealing and multi-step partial dissolution analysis for improved precision and accuracy of zircon ages. Chem Geol 220:47-66. https://doi.org/10.1016/j.chemgeo.2005. 03.011

Mazaheri-Johari M, Kustatscher E, Roghi G, Ghasemi-Nejad E, Gianolla $P$ (2021) A monotypic stand of Neocalamites iranensis $n$. sp. from the Carnian Pluvial Episode (Late Triassic) of the Aghdarband area, NE Iran (Turan Plate). Riv Ital Paleontol Stratigr 127:189-209

Mazaheri-Johari M, Roghi G, Caggiati M, Kustatscher E, GhasemiNejad E, Zanchi A, Gianolla P (2022) Disentangling climate signal from tectonic forcing: the Triassic Aghdarband Basin (Turan Domain, Iran). Palaeogeogr Palaeoclimatol Palaeoecol 586:110777. https://doi.org/10.1016/j.palaeo.2021.110777

Mirnejad H, Lalonde AE, Obeid M, Hassanzadeh J (2013) Geochemistry and petrogenesis of Mashhad granitoids: an insight into the geodynamic history of the Paleo-Tethys in Northeast of Iran. Lithos 170:105-116. https://doi.org/10.1016/j.lithos.2013.03.003

Mohammadi A, Burg JP, Bouilhol P, Ruh J (2016) U-Pb geochronology and geochemistry of Zahedan and Shah Kuh plutons, southeast Iran: implication for closure of the South Sistan suture zone. Lithos 248-251:293-308. https://doi.org/10.1016/j.lithos.2016. 02.003

Muttoni G, Mattei M, Balini M, Zanchi A, Gaetani M, Berra F (2009) The drift history of Iran from the Ordovician to the Triassic. Geol Soc Lond Spec Publ 312:7-29

Natal'in BA, Sengör AMC (2005) Late Palaeozoic to Triassic evolution of the Turan and Scythian platforms: the pre-history of the Palaeo-Tethyan closure. Tectonophys 404:175-202

Omidianfar S, Monsef I, Rahgoshay M, Zheng J, Cousens B (2020) The middle Eocene high-K magmatism in Eastern Iran Magmatic Belt: constraints from $\mathrm{U}-\mathrm{Pb}$ zircon geochronology and $\mathrm{Sr}-\mathrm{Nd}$ isotopic ratios. Int Geol Rev 62:1751-1768. https://doi.org/10.1080/00206 814.2020.1716272

Pang KN, Chung SL, Zarrinkoub MH, Khatib MM, Mohammadi SS, Chiu HY, Chu CH, Lee HY, Lo CH (2013) Eocene-Oligocene post-collisional magmatism in the Lut-Sistan region, eastern Iran: magma genesis and tectonic implications. Lithos 180-181:234 251. https://doi.org/10.1016/j.lithos.2013.05.009

Pearce J (2008) Geochemical fingerprinting of oceanic basalts with applications to ophiolite classification and the search for Archean oceanic crust. Lithos 100:14-48

Pearce JA, Harris NW, Tindle AG (1984) Trace element discrimination diagrams for the tectonic interpretation of granitic rocks. J Petrol 25:956-983

Peccerillo AB, Taylor SR (1976) Geochemistry of Eocene calc-alkaline volcanic rocks from the Kastamonu area, Northern Turkey. Contrib Mineral Petrol 58:63-81. https://doi.org/10.1007/BF003 84745

Pirnia T, Saccani E, Torabi G, Chiari M, Goričan Š, Barbero E (2020) Cretaceous tectonic evolution of the Neo-Tethys in Central Iran: evidence from petrology and age of the Nain-Ashin ophiolitic basalts. Geosci Front 11:57-81. https://doi.org/10.1016/j.gsf. 2019.02.008 
Ramezani J, Tucker RD (2003) The Saghand Region, central Iran: U-Pb geochronology, petrogenesis and implications for Gondwana tectonics. Am J Sci 303:622-665. https://doi.org/10.2475/ajs.303.7. 622

Ranjbar Moghadam F, Masoudi F, Corfu F, Homam SM (2018) Ordovician mafic magmatism in an Ediacaran arc complex, Sibak, NE Iran: the eastern tip of the Rheic Ocean. Can J Earth Sci 55:11731182. https://doi.org/10.1139/cjes-2018-0072

Rezaei-Kahkhaei M, Corfu F, Sheykhi M, Ghasemi H, Shi Y (2021) Mineral chemistry and ages of the Eocene Gapdan granitoid pluton and related dykes (Sistan suture zone, eastern Iran): multistage emplacement of a zoned pluton during progressive deformation and exhumation. J Asian Earth Sci 216:104813. https://doi. org/10.1016/j.jseaes.2021.104813

Rossetti F, Nasrabady M, Vignaroli G, Theye T, Gerdes A, Razavi SMH, Moinvaziri H (2010) Early Cretaceous migmatitic mafic granulites from the Sabzevar range (NE Iran): implications for the closure of the Mesozoic peri-Tethyan oceans in central Iran. Terra Nova 22:26-34. https://doi.org/10.1111/j.1365-3121.2009.00912.x

Rossetti F, Nasrabady M, Theye T, Gerdes A, Monié P, Lucci F, Vignaroli G (2014) Adakite differentiation and emplacement in a subduction channel: the late Paleocene Sabzevar magmatism (NE Iran). Geol Soc Am Bull 126:317-343. https://doi.org/10.1130/B30913.1

Ruttner AW (1993) Southern borderland of Triassic Laurasia in north-east Iran. Geol Rundsch 82:110-120

Schärer U (1984) The effect of initial ${ }^{230} \mathrm{Th}$ disequilibrium on young U-Pb ages: the Makalu case, Himalaya. Earth Planet Sci Lett 67:191-204

Sengör AMC (1984) The Cimmeride orogenic system and the tectonic of Eurasia. Geol Soc Am Spec Pap 195:82

Shafaii Moghadam HS, Corfu F, Chiaradia M, Stern RJ, Ghorbani G (2014) Sabzevar Ophiolite, NE Iran: progress from embryonic oceanic lithosphere into magmatic arc constrained by new isotopic and geochemical data. Lithos 210:224-241. https://doi.org/10.1016/j. lithos.2014.10.004

Shafaii Moghadam HS, Li X-H, Ling X-X, Stern RJ, Khedr MZ, Chiaradia M, Ghorbani G, Arai S, Tamura A (2015a) Devonian to Permian evolution of the Paleo-Tethys Ocean: new evidence from $\mathrm{U}-\mathrm{Pb}$ zircon dating and $\mathrm{Sr}-\mathrm{Nd}-\mathrm{Pb}$ isotopes of the Darrehanjir-Mashhad “ophiolites", NE Iran. Gond Res 28:781-799. https://doi.org/10. 1016/j.gr.2014.06.009

Shafaii Moghadam HS, Li X-H, Ling X-X, Santos JF, Stern RJ, Li Q-L, Ghorbani G (2015b) Eocene Kashmar granitoids (NE Iran): petrogenetic constraints from $\mathrm{U}-\mathrm{Pb}$ zircon geochronology and isotope geochemistry. Lithos 216-217:118-135

Shafaroudi AM, KarimpourGolmohammadi MHA (2013) Zircon U-Pb geochronology and petrology of intrusive rocks in the C-North and Baghak districts, Sangan iron mine, NE Iran. J Asian Earth Sci 64:256-271

Shahbazi H, Sepahi AA, Shakouri MA (2021) Zircon U-Pb ages and petrogenesis of the middle Eocene Aliabad Daman pluton, Northeast Iran: implications for magmatic activity along the Doruneh fault zone. Arab J Geosci 14:212. https://doi.org/10.1007/ s12517-020-06437-w

Shand SJ (1947) The eruptive rocks. Wiley, New York, p 444

Sheikholeslami MR, Kouhpeyma M (2012) Structural analysis and tectonic evolution of the eastern Binalud Mountains, NE Iran. J Geodyn 61:23-46

Siehl A (2017) Structural setting and evolution of the Afghan orogenic segment-a review. In: Brunet M-F, McCann T, Sobel ER (eds) Geological evolution of Central Asian Basins and the Western Tien Shan Range, vol 427. Springer, London, pp 57-88. https://doi.org/ $10.1144 / \mathrm{SP} 427.8$

Stampfli GM, Borel GD (2002) A plate tectonic model for the Paleozoic and Mesozoic constrained by dynamic plate boundaries and restored synthetic oceanic isochrons. Earth Planet Sci Lett 196:17-33
Stampfli GM, Hochard C, Vérard C, Wilhem C, von Raumer J (2013) The formation of Pangea. Tectonophysics 593:1-19. https://doi.org/10. 1016/j.tecto.2013.02.037

Stöcklin J (1977) Structural correlation of the Alpine ranges between Iran and Central Asia. Mém Hors Série Soc Géol France 8:333-353

Sun S-S, McDonough WF (1989) Chemical and isotopic systematic of oceanic basalts: implications for mantle composition and processes. Geol Soc Lond Spec Publ 42:313-345. https://doi.org/10.1144/GSL. SP.1989.042.01.19

Tadayon M, Rossetti F, Zattin M, Nozaem R, Calzolari G, Madanipour S, Salvini F (2017) The post-Eocene evolution of the Doruneh Fault region (Central Iran): the intraplate response to the reorganization of the Arabia-Eurasia collision zone. Tectonics 36:3038-3064. https:// doi.org/10.1002/2017TC004595

Tadayon M, Rossetti F, Zattin M, Calzolari G, Nozaem R, Salvini F, Faccenna C, Khodabakhshi P (2019) The long-term evolution of the Doruneh Fault region (Central Iran): a key to understanding the spatio-temporal tectonic evolution in the hinterland of the Zagros convergence zone. Geol J 54:1454-1479. https://doi.org/10.1002/ gj. 3241

Taylor SR, McLennan SM (1985) The continental crust: its composition and evolution. Blackwell Scientific Publications, Oxford, pp 1-328

Ternet Y, Guillou Y, Maurizot P (1980) Geological map of Iran, 1:100 000 series, Sheet 8059 Khaf

Tischendorf G, Gottesmann B, Förster HJ, Trumbull RB (1997) On Libearing micas: estimating $\mathrm{Li}$ from electron microprobe analyses and an improved diagram for graphical representation. Mineral Mag 61:809-834

Topuz G, Hegner E, Homam SM, Ackerman L, Pfänder JA, Karimi H (2018) Geochemical and geochronological evidence for a Middle Permian oceanic plateau fragment in the Paleo-Tethyan suture zone of NE Iran. Contrib Mineral Petrol 173:81. https://doi.org/10.1007/ s00410-018-1506-x

Torsvik TH, Cocks LRM (2013) Gondwana from top to base in space and time. Gond Res 24:999-1030. https://doi.org/10.1016/j.gr.2013. 06.012

Uchida E, Endo S, Makino M (2007) Relationship between solidification depth of granitic rocks and formation of hydrothermal ore deposits. Resour Geol 57:47-56

Wilmsen M, Fürsich FT, Taheri J (2009) The Shemshak Group (LowerMiddle Jurassic) of the Binalud Mountains, NE Iran: stratigraphy, depositional environments and geodynamic implications. In: Brunet M-F, Wilmsen M, Granath JW (eds) South Caspian to Central Iran Basins, vol 312. Geological Society, London, Special Publications, Berlin, pp 175-188

Zanchetta S, Zanchi A, Villa IM, Poli S, Muttoni G (2009) The Shanderman eclogites: a Late Carboniferous high-pressure event in the NW Talesh Mountains (NW Iran). In: Brunet M-F, Wilmsen M, Granath JW (eds) South Caspian to Central Iran Basins, vol 312. Geological Society, London, Special Publications, London, pp 57-78

Zanchetta S, Berra F, Zanchi A, Bergomi M, Caridroit M, Nicora A, Heidarzadeh G (2013) The record of the Late Paleozoic active margin of the Palaeotethys in NE Iran: constraints on the Cimmerian orogeny. Gond Res 24:1237-1266. https://doi.org/10.1016/j.gr.2013.02.013

Zanchi A, Zanchetta S, Berra F, Mattei M, Garzanti E, Molyneux S, Nawab A, Sabouri J (2009a) The Eo-Cimmerian (Late? Triassic) orogeny in North Iran. In: Brunet M-F, Wilmsen M, Granath JW (eds) South Caspian to Central Iran Basins, vol 312. Geological Society, London, Special Publications, London, pp 31-56

Zanchi A, Zanchetta S, Garzanti E, Balin M, Berra F, Mattei M, Muttoni G (2009b) The Cimmerian evolution of the Nakhlak-Anarak area, central Iran, and its bearing for the reconstruction of the history of the Eurasian margin. In: Brunet M-F, Wilmsen M, Granath JW (eds) South Caspian to Central Iran Basins, vol 312. Geological Society, London, Special Publications, London, pp 261-286 
Zanchi A, Malaspina N, Zanchetta S, Berra F, Benciolini L, Bergomi M, Cavallo A, Javadi HR, Kouhpeyma M (2015) The Cimmerian accretionary wedge of Anarak, Central Iran. J Asian Earth Sci 102:45-72. https://doi.org/10.1016/j.jseaes.2014.08.030

Zanchi A, Zanchetta S, Balini M, Ghassemi MR (2016) Oblique convergence during the Cimmerian collision: evidence from the Triassic Aghdarband Basin, NE Iran. Gondw Res 38:149-170. https://doi. org/10.1016/j.gr.2015.11.008
Zanchi A, Zanchetta S, Berra F, Mattei M, Javadi HR, Montemagni C (2021) Cenozoic dextral shearing along the Arusan sector of the Great Kavir-Doruneh Fault System (Central Iran). Tectonics 40:e2021TC006766. https://doi.org/10.1029/2021TC006766

Zarrinkoub MH, Pang K-N, Chung S-L, Khatib MM, Mohammadi SS, Chiu HY, Lee H-Y (2012) Zircon U-Pb age and geochemical constraints on the origin of the Birjand ophiolite, Sistan suture zone, eastern Iran. Lithos 154:392-405 